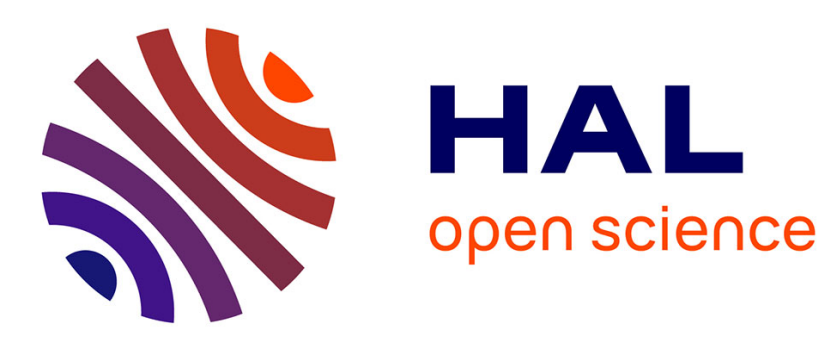

\title{
Numerical bifurcation analysis for 3-dimensional sudden expansion fluid dynamic problem
}

Yann Guevel, Tual Allain, Grégory Girault, Jean-Marc Cadou

\section{To cite this version:}

Yann Guevel, Tual Allain, Grégory Girault, Jean-Marc Cadou. Numerical bifurcation analysis for 3dimensional sudden expansion fluid dynamic problem. International Journal for Numerical Methods in Fluids, 2018, 87 (1), pp.1-26. 10.1002/fld.4478 . hal-01807605

\section{HAL Id: hal-01807605 https://hal.science/hal-01807605}

Submitted on 30 Jun 2018

HAL is a multi-disciplinary open access archive for the deposit and dissemination of scientific research documents, whether they are published or not. The documents may come from teaching and research institutions in France or abroad, or from public or private research centers.
L'archive ouverte pluridisciplinaire HAL, est destinée au dépôt et à la diffusion de documents scientifiques de niveau recherche, publiés ou non, émanant des établissements d'enseignement et de recherche français ou étrangers, des laboratoires publics ou privés. 


\title{
Numerical bifurcation analysis for 3-dimensional sudden expansion fluid dynamic problem
}

\author{
Y. Guevel $^{1} \quad$ T. Allain ${ }^{1} \quad$ G. Girault ${ }^{1,2} \quad$ J.M. Cadou ${ }^{1}$ \\ ${ }^{1}$ Univ. Bretagne Sud, FRE CNRS 3744, \\ IRDL, Lorient, France \\ ${ }^{2}$ Centre de Recherche, Ecoles de Saint-Cyr \\ Coëtquidan, Guer, France
}

Summary This paper deals with bifurcation analysis methods based on the asymptotic-numerical method. It is used to investigate 3-dimensional (3D) instabilities in a sudden expansion. To do so, highperformance computing is implemented in ELMER, ie, an open-source multiphysical software. In this work, velocity-pressure mixed vectors are used with asymptotic-numerical method-based methods, remarks are made for the branch-switching method in the case of symmetry-breaking bifurcation, and new 3D instability results are presented for the sudden expansion ratio, ie, $E=3$. Critical Reynolds numbers for primary bifurcations are studied with the evolution of a geometric parame-ter. New values are computed, which reveal new trends that complete a previous work. Several kinds of bifurcation are depicted and tracked with the evolution of the spanwise aspect ratio. One of these relies on a fully 3D effect as it breaks both spanwise and top-bottom symmetries. This bifurcation is found for smaller aspect ratios than expected. Furthermore, a critical Reynolds number is found for the aspect ratio, ie, $A_{i}=1$, which was not previously reported. Finally, pri-mary and secondary bifurcations are efficiently detected and all post-bifurcated branches are followed. This makes it possible to plot a complete bifurcation diagram for this $3 \mathrm{D}$ case.

Keywords asymptotic-numerical method, branch switching, continuation, high-performance computing, Navier-Stokes, nonlinear solvers, numerical bifurcation analysis, sudden expansion, 3-dimensional flow

\section{1 | INTRODUCTION}

Numerical bifurcation analysis of flow problems governed by the discretized Navier-Stokes equations with a large number of degrees of freedom is still a challenging task. A review of the existing numerical bifurcation methods in fluid dynamics was proposed in the work of Dijkstra et al. ${ }^{1}$ Toolbox exists for ordinary differential equations, such as MatCont ${ }^{2}$ and AUTO-07p, ${ }^{3}$ hence with a small number of degrees of freedom. ${ }^{4} \mathrm{~A}$ large-scale toolbox for systems of discretized partial differential equations is the library of continuation algorithms LOCA ${ }^{5}$ as part of the Trilinos framework. ${ }^{6}$

An alternative to the well-known incremental-iterative methods ${ }^{7-9}$ is the asymptotic-numerical method (ANM). ${ }^{10}$ This ANM is the association of a perturbation technique and a spatial discretization method, in our case the finite element method (FEM). It has been successfully applied in nonlinear solid mechanics ${ }^{11,12}$ or in fluid mechanics. ${ }^{13-15}$ The main advantage of the ANM is its ability to determine analytical nonlinear solutions ${ }^{16,17}$ with computational CPU time lower 
than with that of the classical incremental-iterative method. On these nonlinear solutions, some bifurcation indicators have been introduced. ${ }^{12,18-20}$ High-performance computation based on the ANM for flow problems was proposed in the work of Medale and Cochelin ${ }^{21}$ as an in-house code.

Parametric studies were proposed for the 3-dimensional (3D) sudden expansion in other works. ${ }^{22-25}$ Numerical bifurcation analysis using the aspect ratio $E=3$ is available in other works, ${ }^{26-29}$ but only the first primary bifurcation was investigated. More recently, a new powerful feature of the ANM, ${ }^{12}$ which makes it possible to enhance the detection and continuation in the vicinity of a simple bifurcation, has been applied to study additional primary bifurcation for several spanwise aspect ratio. ${ }^{14}$

In this work, we propose to complete previous studies by tracking each kind of primary bifurcation when the aspect ratio evolves. Moreover, secondary bifurcations are investigated to plot a complete bifurcation diagram. Furthermore, new information are given for the spanwise symmetry-breaking bifurcation described in the works of Medale and Cochelin ${ }^{14}$ and Chiang et al. ${ }^{27}$ Regarding the ANM-based method, remarks are made for the branch-switching technique in the case

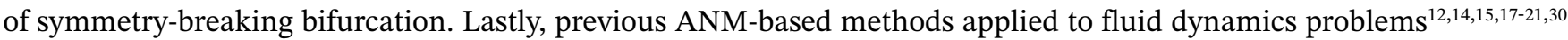
made no use of pressure information because static condensation was used. In this work, full velocity-pressure mixed vectors are used in the ANM-based methods, such that flows and specific vectors are evaluated with pressure information.

This paper is organized as follows. The numerical methods are described in the first part. Implementation of the ANM in ELMER is briefly mentioned in the second part. In the third part, bifurcation analysis is proposed for 3D flow in a sudden expansion. Within this part, information of interest are compared to the literature, the evolution of the first primary bifurcation is investigated, and bifurcation modes are depicted with velocity and pressure components. Lastly, a bifurcation diagram is proposed to study secondary bifurcations.

\section{2 | NUMERICAL METHODS}

\section{1 | Governing equations}

The steady Navier-Stokes equations for Newtonian and incompressible fluid in a domain $(\Omega)$ are as follows:

$$
\begin{aligned}
-2 \mu \operatorname{div} \mathbf{D}(\mathbf{u})+\operatorname{grad} p+\rho \mathbf{u} \cdot \operatorname{grad} \mathbf{u} & =\mathbf{0} \text { in }(\Omega) \\
\operatorname{div} \mathbf{u} & =0 \text { in }(\Omega) \\
\mathbf{u} & =\lambda \mathbf{u}_{d} \text { on }(\Gamma),
\end{aligned}
$$

with $\mathbf{u}$ and $\mathrm{p}$ being the velocity and pressure unknowns, respectively, and $\lambda$ being a control parameter. A generic velocity profile $\mathbf{u}_{d}$ is prescribed on the boundary $(\Gamma)$. $\mathbf{D}(\mathbf{u})$ is the symmetric part of the velocity gradient tensor. Both density $\rho$

and kinematic viscosity $\mu$ are constant in the fluid domain. The Reynolds number is defined as $\operatorname{Re}=\lambda \frac{\rho \mathbf{u}_{d}^{\max } h}{\mu}$, with $h$ being a characteristic length of the model.

The weak form of the Navier-Stokes equations can be defined according to

$$
\mathrm{L}(\mathbf{U})+\mathrm{Q}(\mathbf{U}, \mathbf{U})=\lambda \mathbf{F} \quad \text { in }(\Omega)
$$

where the linear operator $\mathrm{L}(\bullet)$ contains the diffusion, the pressure gradient, and the continuity terms. The nonlinear operator Q $(\bullet, \bullet)$ contains the convection terms. Dirichlet boundary condition Equation (3) produces the right-hand-side (RHS) term, which is equivalent to a fictitious force. ${ }^{16,17}$

\section{2 | Continuation with perturbation method}

Pseudo-arc-length path-following technique ${ }^{16}$ based on the ANM is used to perform the continuation of the steady flow solutions. This has been successfully implemented for 2-dimensional (2D) flows in the case of steady incompressible Navier-Stokes equations in other works ${ }^{12,17-19}$ and for non-Newtonian fluids in the work of Jawadi et al. ${ }^{20}$ Recently, 3D flow study has been proposed in the work of Medale and Cochelin. ${ }^{14}$ 
The ANM relies on a linearization technique where the unknowns of the nonlinear problem are sought as power series as follows:

$$
\mathbf{X}(a)=\mathbf{X}_{0}+\sum_{i=1}^{N} a^{i} \mathbf{X}_{i},
$$

where $\mathbf{X}_{0}=\left\{\mathbf{U}_{0} \lambda_{0}\right\}^{\top}$ is a known regular solution with the mixed vector notation $\mathbf{U}_{0}=\left\{\mathbf{u}_{0} p_{0}\right\}^{\top}, a \in \mathbb{R}$ is a perturbation parameter, and $N$ is the truncation order of this polynomial representation.

Although several definitions exist, the perturbation path parameter is chosen as follows:

$$
a=\left\langle\mathbf{u}-\mathbf{u}_{0}\right\rangle \mathbf{u}_{1}+\left(\lambda-\lambda_{0}\right) \lambda_{1},
$$

where $\langle\bullet, \bullet\rangle$ is the classic Euclidean dot product. Introducing Equation (5) in the nonlinear system Equation (1) and equating like powers of $a$, a set of linear algebraic systems is obtained. The same tangent operator is used for each of those linear systems and is defined as follows:

$$
\mathrm{L}_{t}^{0}(\bullet)=\mathrm{L}(\bullet)+\mathrm{Q}\left(\mathbf{U}_{0}, \bullet\right)+\mathrm{Q}\left(\bullet, \mathbf{U}_{0}\right)
$$

The first order reads

$$
\begin{aligned}
\mathrm{L}_{t}^{0}\left(\mathbf{U}_{1}\right) & =\lambda_{1} \mathbf{F} \\
\left\langle\mathbf{u}_{1}, \mathbf{u}_{1}\right\rangle+\lambda_{1}^{2} & =1,
\end{aligned}
$$

and the recurrence relation for $k \in[2, N]$ is

$$
\begin{aligned}
\mathrm{L}_{t}^{0}\left(\mathbf{U}_{k}\right) & =\lambda_{k} \mathbf{F}-\sum_{i=1}^{k-1} \mathbf{Q}\left(\mathbf{U}_{i}, \mathbf{U}_{k-i}\right) \\
\left\langle\mathbf{u}_{k}, \mathbf{u}_{1}\right\rangle+\lambda_{k} \lambda_{1} & =0 .
\end{aligned}
$$

Once the series $\left\{\mathbf{U}_{i}, \lambda_{i}\right\}_{i=1, N}$ are computed using Equation (8)-(9), small part of the steady flow solution branch is approximated continuously with the polynomial representation Equation (5).

The range of validity of this approximation is defined as follows:

$$
a_{\text {maxpoly }}=\left(\eta \frac{\left\|\mathbf{U}_{1}\right\|}{\left\|\mathbf{U}_{N}\right\|}\right)^{1 /(N-1)},
$$

with $\eta$ being a user-defined tolerance. Other definitions may be used. ${ }^{16}$

Finally, the ANM requires 2 user parameters, ie, the first one being the truncation order $N$ and the second one is the tolerance parameter $\eta$. The latter governs the accuracy of the computed nonlinear predictions. Lastly, new regular solution $\mathbf{X}_{0}=\mathbf{X}\left(a_{\max }\right)$ is evaluated with Equation (5) and new continuation step might be performed. We recall that the approximation of the flow solution is continuous in a path-following continuation step. This makes it possible to evaluate steady flow solutions at an exact Reynolds number.

Usually, the ANM nonlinear prediction does not need correction at the end of a step. Nevertheless, for some values of the chosen parameter $\eta$, the accuracy of the solution obtained with the ANM cannot be satisfactory. It means that the computed residual is greater than a given accuracy. In such a case, very efficient and cheaper correctors can be used at the end of the continuation step to improve the quality of the ANM solution (see, for example, the work of Cadou and Potier-Ferry ${ }^{31}$ ).

\section{3 | Steady bifurcation detection}

Once steady nonlinear solution branch has been computed, it is worth determining the critical Reynolds numbers for which a steady bifurcation appears. These singularities often characterize the loss of uniqueness of the flow solution. In this study, only simple symmetry-breaking bifurcations of steady flow are considered. Nevertheless, these techniques might be applied for other kinds of bifurcation analysis. ${ }^{11,21}$

Van Dyke ${ }^{32}$ showed that power series analysis may reveal some features about singularity in its range of validity. Thus, the ANM series analysis has been proposed for steady bifurcation detection in the work of Cochelin and Medale. ${ }^{12}$ It has 
been applied to Newtonian incompressible fluid flows in the works of Cochelin and Medale ${ }^{12,14}$ and for non-Newtonian fluids in the work of Jawadi et al. ${ }^{20}$ It is demonstrated in the work of Cochelin and Medale ${ }^{12}$ that the pseudo-arc-length continuation is perturbed in the neighborhood of a singularity. Geometric progression appears in the ANM series in the vicinity of a steady bifurcation point. The power series $\left\{\mathbf{X}_{i}\right\}$ computed in the singularity vicinity using Equation (8)-(9) is, in fact, a linear combination of a geometric progression series with a nearly flawless ones as follows:

$$
\left\{\mathbf{X}_{i}\right\}=\left\{\hat{\mathbf{X}}_{i}\right\}_{\text {flawless }}+\left\{\mathbf{X}_{i}\right\}_{\text {geometric }}, \quad \text { for } i=1, N .
$$

Determining a steady bifurcation consists in finding a geometric progression into the polynomial representation Equation (5). This is numerically detected via collinearity condition and relative error test applied to the last terms of the series as follows:

$$
\begin{gathered}
\sum_{p=N-3}^{N-2}\left(\frac{\left|\alpha_{p}\right|^{1 /(N-p)}}{\left|\alpha_{n-1}\right|}-1\right)^{2}<\epsilon_{g p 1}, \quad \text { with } \quad \alpha_{p}=\frac{\left\langle\mathbf{X}_{p}, \mathbf{X}_{N}\right\rangle}{\left\langle\mathbf{X}_{N}, \mathbf{X}_{N}\right\rangle} \\
\sum_{p=N-3}^{N-1}\left(\left\|\mathbf{X}_{p}^{\perp}\right\| /\left\|\mathbf{X}_{p}\right\|\right)<\epsilon_{g p 2}, \quad \text { with } \quad \mathbf{X}_{p}^{\perp}=\mathbf{X}_{p}-\alpha_{p} \mathbf{X}_{N} .
\end{gathered}
$$

The parameters $\epsilon_{g p 1}=10^{-6}$ and $\epsilon_{g p 2}=10^{-3}$ gave satisfactory results for steady bifurcation detection in this study. It should be noted that a large truncation order, around $N=30$ in this study, is required to accurately detect the geometric progression. Once the common ratio $1 / \alpha_{c}=1 / \alpha_{N-1}$ and the associated scale factor $\alpha_{c}^{N} \mathbf{X}_{N}$ are determined, a nearly flawless enhanced series is computed as follows:

$$
\widehat{\mathbf{X}}_{i}=\mathbf{X}_{i}-\alpha_{c}^{N-i} \mathbf{X}_{N}, \quad \text { for } i=1, N
$$

In the work of Cochelin and Medale, ${ }^{12}$ it is demonstrated that $\alpha_{c}$ is exactly the arc-length distance to the critical point. Thus, the flow solution at this singular point is computed using the flawless enhanced series as follows:

$$
\mathbf{X}_{c}=\mathbf{X}\left(a=\alpha_{c}\right)=\mathbf{X}_{0}+\sum_{i=1}^{N-1} \alpha_{c}^{i} \widehat{\mathbf{X}}_{i}
$$

This constitutes an easy method to detect accurately critical solution because it is done while performing continuation steps with no additional computational effort.

\section{4 | Branch switching at simple bifurcation point}

At simple steady bifurcation point, families of flow solution branches might be found using the classical ANM path-following technique by varying the ANM user parameters $N$ and $\eta \cdot{ }^{17}$ It requires a lot of computations with no possible guess of which parameters lead to a specific branch. Guevel et $\mathrm{al}^{19}$ proposed a branch-switching method for the Navier-Stokes equations resolution. Then, approximations of nonlinear post-bifurcated branches emanating from the singular solution at a simple bifurcation point are computed using an adapted ANM continuation. This branch-switching technique relies on the traditional bifurcation theory. ${ }^{8,9,33,34}$ It has been successfully coupled with the ANM in other works ${ }^{11,12,35,36}$ in the solid mechanics framework. Because FEM operators in fluid mechanics problems are unsymmetric, an adaptation is proposed in the works of Medale and Cochelin ${ }^{14}$ and Guevel et al. ${ }^{19}$

First, tangents of the 2 intersecting branches at a simple bifurcation point are determined. By choosing one of those tangents, it makes it possible to switch from a branch to another. Those nonlinear post-bifurcated branches are computed with a modified ANM path-following technique as presented in the work of Guevel et al. ${ }^{19}$ Hence, the nonlinear post-bifurcated branches are sought as a power series representation as follows:

$$
\mathbf{X}^{b i}(a)=\mathbf{X}_{c}+\sum_{j=1}^{N} a^{j} \mathbf{X}_{j}^{b i}, \quad \text { for } i=1,2 .
$$

The starting point is the singular solution $\mathbf{X}_{c}$ computed as in Equation (15). Injecting the polynomial representation Equation (16) in the nonlinear system Equation (1), a set of linear algebraic systems is obtained equating like powers of $a$. The tangent operator denoted as $\mathrm{L}_{t}^{c}$ is assembled as in Equation (7) with the solution at the bifurcation point. As it is singular, a special care is required to compute the post-bifurcated polynomial representation using this operator. Thus, tangents at the bifurcation point are the first term of this representation. 
At a simple bifurcation point, the tangent operator has the following properties:

$$
\begin{aligned}
\operatorname{Ker}\left(\mathrm{L}_{t}^{c}\right) & =\{\boldsymbol{\Phi}\}, \quad \boldsymbol{\Phi} \in \Omega,\langle\boldsymbol{\Phi}, \boldsymbol{\Phi}\rangle=1 \\
\operatorname{Ker}\left(\mathrm{L}_{t}^{c \top}\right) & =\{\boldsymbol{\Psi}\}, \quad \boldsymbol{\Psi} \in \Omega,\langle\boldsymbol{\Psi}, \boldsymbol{\Phi}\rangle=1,
\end{aligned}
$$

with $\Phi$ being the so-called bifurcation mode and $\boldsymbol{\Psi}$ being the associated left bifurcation mode. Moreover, there exists a unique particular solution vector $\mathbf{W}$ such that

$$
\mathrm{L}_{t}^{c}(\mathbf{W})=\mathbf{F}, \quad \mathbf{W} \in \Omega,<\mathbf{W}, \boldsymbol{\Phi}>=0 .
$$

Different strategies are available to determine those vectors. ${ }^{33} \mathrm{~A}$ way to compute those vectors is described in the following. Additionally, all those specific vectors are computed in this work as full velocity-pressure mixed vectors.

\subsection{1 | Tangents determination}

At first order of the path parameter $a$, the tangents $\mathbf{X}_{1}^{b i}=\left\{\begin{array}{l}\mathbf{U}_{1}^{b i} \\ \lambda_{1}^{b i}\end{array}\right\}_{i=1,2}$ are solutions of the following linear system:

$$
\begin{aligned}
\mathrm{L}_{t}^{c}\left(\mathbf{U}_{1}^{b i}\right) & =\lambda_{1} \mathbf{F} \\
\left\langle\mathbf{U}_{1}^{b i}, \mathbf{U}_{1}^{b i}\right\rangle+\left(\lambda_{1}^{b i}\right)^{2} & =1 .
\end{aligned}
$$

The singularity at the bifurcation point is treated classically with the help of the Lyapunov-Schmidt reduction. ${ }^{19,33,37,38}$ The 2 tangents are written as follows:

$$
\mathbf{U}_{1}^{b i}=\lambda_{1}^{b i} \mathbf{W}+\eta_{1}^{b i} \mathbf{\Phi}
$$

with $\lambda_{1}^{b i}, \eta_{1}^{b i} \in \mathbb{R}$ being 2 scalars to be determined. To do so, solve the following linear system defined at the second order:

$$
\begin{aligned}
\mathrm{L}_{t}^{c}\left(\mathbf{U}_{2}^{b i}\right) & =\lambda_{2} \mathbf{F}-\mathbf{Q}\left(\mathbf{U}_{1}^{b i}, \mathbf{U}_{1}^{b i}\right) \\
\left\langle\mathbf{U}_{2}^{b i}, \mathbf{U}_{1}^{b i}\right\rangle+\lambda_{2}^{b i} \lambda_{1}^{b i} & =0 .
\end{aligned}
$$

Projecting Equation (22a) on the left bifurcation mode $\boldsymbol{\Psi}$, Equation (18) leads to the well-known Algebraic Bifurcation Equation (ABE) as follows:

$$
\left\langle\Psi, Q\left(\mathbf{U}_{1}^{b i}, \mathbf{U}_{1}^{b i}\right)\right\rangle=0 .
$$

Using the Lyapunov-Schmidt reduction Equation (21), the following quadratic ABE is obtained:

$$
a_{b}\left(\lambda_{1}^{b i}\right)^{2}+b_{b} \lambda_{1}^{b i} \eta_{1}^{b i}+c_{b}\left(\eta_{1}^{b i}\right)^{2}=0 .
$$

This quadratic equation may be easily solved using Equation (20b), with the following coefficients:

$$
\begin{aligned}
a_{b} & =\langle\Psi, \mathrm{Q}(\mathbf{W}, \mathbf{W})\rangle \\
b_{b} & =\langle\Psi, \widetilde{Q}(\Phi, \mathbf{W})\rangle \\
c_{b} & =\langle\Psi, \mathrm{Q}(\Phi, \Phi)\rangle .
\end{aligned}
$$

In Equation (25b), the operator $\widetilde{\mathrm{Q}}(a, b)$ is defined according to the expression $\widetilde{\mathrm{Q}}(a, b)=\mathrm{Q}(a, b)+\mathrm{Q}(b, a)$. The general case with restriction to $\eta_{1}^{b i} \neq 0$ and $a_{b} \neq 0$ gives

$$
t^{b i}=\frac{\lambda_{1}^{b i}}{\eta_{1}^{b i}}=\frac{-b_{b}+(-1)^{i} \sqrt{b_{b}^{2}-4 a_{b} c_{b}}}{2 a_{b}} .
$$

Using the norm condition Equation (20b) and the property $\langle\mathbf{W}, \boldsymbol{\Phi}\rangle=0$, it is obtained:

$$
\eta_{1}^{b i}=\frac{1}{\sqrt{\left(t^{b i}\right)^{2}\langle\mathbf{W}, \mathbf{W}\rangle+\langle\boldsymbol{\Phi}, \boldsymbol{\Phi}\rangle+\left(t^{b i}\right)^{2}}}
$$

where $\lambda_{1}^{b i}$ is automatically deduced using Equation (26). 


\section{Remark on symmetry-breaking bifurcation case}

Pitchfork bifurcation occurs at simple bifurcation point if flow symmetry is broken for one of the post-bifurcated family branch. Therefore, the coefficients defined in Equation (25a) are checked numerically. If $a_{b}=c_{b}=0$ and $b_{b} \neq 0$, it implies that pitchfork bifurcation occurs at the detected critical point. ${ }^{39}$ One is easily convinced that the condition $c_{b}=0$ is equivalent to the fact that the bifurcation mode $\boldsymbol{\Phi}$ is the solution of the ABE proposed in Equation (23) and that it is one of the sought tangents. ${ }^{9,39}$ Moreover, $a_{b}=0$ implies that the other tangent is then collinear to the particular solution $\mathbf{W}$.

Finally, in the case of pitchfork bifurcation, the 2 tangents are as follows:

$$
\begin{aligned}
& \mathbf{X}_{1}^{b a}=\left\{\begin{array}{l}
\mathbf{U}_{1}^{b a} \\
\lambda_{1}^{b a}
\end{array}\right\}=\left\{\begin{array}{c}
\boldsymbol{\Phi} \\
0
\end{array}\right\} \\
& \mathbf{X}_{1}^{b s}=\left\{\begin{array}{l}
\mathbf{U}_{1}^{b s} \\
\lambda_{1}^{b s}
\end{array}\right\}=\left\{\begin{array}{c}
\lambda_{1}^{b s} \mathbf{W} \\
\lambda_{1}^{b s}
\end{array}\right\}
\end{aligned}
$$

with

$$
\lambda_{1}^{b s}=\frac{1}{\sqrt{\langle\mathbf{W}, \mathbf{W}\rangle+1}}
$$

\subsection{2 | Specific vectors computation}

In order to evaluate the coefficients in Equation (25) or directly the pitchfork bifurcation tangents as in Equation (29)-(28), the specific vectors $\boldsymbol{\Phi}, \mathbf{W}$, and $\boldsymbol{\Psi}$ are required.

In other works, ${ }^{18,19,35}$ the bifurcation mode $\Phi$ is computed using a classic ANM linear stability analysis technique known as the "bifurcation indicator". It should be noted that this requires some computational efforts. Then, only $\mathbf{W}$ and $\Psi$ remain to be computed using the definitions Equation (18)-(19) by the mean of augmented systems presented in the following. This is done with no assumption of the symmetry-breaking simple bifurcation case.

The recent development proposed in the works of Cochelin and Medale ${ }^{12,14}$ does not use the same Lyapunov-Schmidt reduction. Nevertheless, in the case of the symmetry-breaking bifurcations, vectors $\boldsymbol{\Phi}$ and $\mathbf{W}$ can be computed. The first tangent at the bifurcation point is obtained by differentiating the actual flawless enhanced power series Equation (16) at the critical point as follows:

$$
\mathbf{X}_{1}^{b 1}=\left.\frac{d \mathbf{X}(a)}{d a}\right|_{a=\alpha_{c}}=\sum_{i=1}^{N-1} i \alpha_{c}^{i-1} \widehat{\mathbf{X}}_{i} .
$$

A Gram-Schmidt technique is used to extract the orthogonal vector from the last term of the series as follows:

$$
\mathbf{X}_{1}^{b 1^{\perp}}=\mathbf{X}_{N}-\frac{\left\langle\mathbf{X}_{N}, \mathbf{X}_{1}^{b 1}\right\rangle}{\left\langle\mathbf{X}_{1}^{b 1}, \mathbf{X}_{1}^{b 1}\right\rangle} \mathbf{X}_{1}^{b 1} .
$$

In the case of symmetric branch, the following equality holds: $\mathbf{\Phi}=\mathbf{X}_{1}^{b 1^{\perp}}$. Contrariwise, if the continuation is performed on a post-bifurcated solution, from an antisymmetric state, then the bifurcation mode is the tangent computed as $\mathbf{\Phi}=\mathbf{X}_{1}^{b 1}$.

Moreover, the particular solution W might be computed using Equation (31) or Equation (32) because of the orthogonality relation. Instead, it is used as an augmented system, which makes it possible to compute $\mathbf{W}$ and is reused in the following to circumvent the singularity of the tangent operator. This does not require more computational effort. The vector $\mathbf{W}$, which is the solution of the linear equation in Equation (19), is orthogonal to the bifurcation mode $\boldsymbol{\Phi}$ so that the augmented system to be solved is written as follows:

$$
\left[\begin{array}{cc}
L_{t}^{c} & \boldsymbol{\Phi} \\
\boldsymbol{\Phi}^{\top} & 0
\end{array}\right]\left\{\begin{array}{c}
\mathbf{W} \\
\kappa
\end{array}\right\}=\left\{\begin{array}{l}
\mathbf{F} \\
0
\end{array}\right\}
$$

where $\kappa$ acts as a Lagrangian multiplier, which would be as small as possible.

In the case of the Navier-Stokes equations, the left mode remains to be computed using either an augmented system, ${ }^{19}$ or with iterative techniques such as bordering technique as presented in the work of Cochelin and Medale, ${ }^{12}$ or with an inverse power method. ${ }^{14}$ Then, using the same consideration for the left bifurcation mode $\boldsymbol{\Psi}$ (see Equation (18)), the augmented system is written as follows:

$$
\left[\begin{array}{ll}
L_{t}^{c^{\top}} & \boldsymbol{\Phi} \\
\boldsymbol{\Phi}^{\top} & 0
\end{array}\right]\left\{\begin{array}{l}
\boldsymbol{\Psi} \\
\kappa
\end{array}\right\}=\left\{\begin{array}{l}
\mathbf{0} \\
1
\end{array}\right\}
$$


It is noticed that this augmented matrix is the transpose of the precedent one in Equation (33). Specific options might be used in linear solvers to reuse the already factorized matrix Equation (33). In this way, only one factorization is required to compute the specific vectors and for every linear system required for the post-bifurcated branches as described in the following.

\subsection{3 | Post-bifurcated branch continuation}

Once tangents are determined as solutions of Equation (20), high-order terms of the power series Equation (16) are determined for each of the post-bifurcated branches as solution of

$$
\begin{aligned}
\mathrm{L}_{t}^{c}\left(\mathbf{U}_{k}^{b i}\right) & =\lambda_{k}^{b i} \mathbf{F}-\sum_{j=1}^{k-1} \mathbf{Q}\left(\mathbf{U}_{j}^{b i}, \mathbf{U}_{k-j}^{b i}\right) \\
\left\langle\mathbf{U}_{k}^{b i}, \mathbf{U}_{1}^{b i}\right\rangle+\lambda_{k}^{b i} \lambda_{1}^{b i} & =0 .
\end{aligned}
$$

The following Lyapunov-Schmidt reduction ${ }^{12,19}$ is used:

$$
\mathbf{U}_{k}^{b i}=\lambda_{k}^{b i} \mathbf{W}+\eta_{k}^{b i} \mathbf{\Phi}+\mathbf{V}_{k}^{b i}
$$

Injecting Equation (36) in Equation (35), the new vector $\mathbf{V}_{k}^{b i}$ is solution of

$$
\begin{aligned}
\mathrm{L}_{t}^{c}\left(\mathbf{V}_{k}^{b i}\right) & =-\sum_{j=1}^{k-1} \mathbf{Q}\left(\mathbf{U}_{j}^{b i}, \mathbf{U}_{k-j}^{b i}\right) \\
\left\langle\mathbf{V}_{k}^{b i}, \boldsymbol{\Phi}\right\rangle & =0 .
\end{aligned}
$$

This system of equations is exactly the linear augmented system described in Equation (33), with different RHS vector. It makes it possible to compute $\mathbf{V}_{k}^{b i}$ in an efficient way because only a new RHS needs to be assembled.

The coefficients $\lambda_{k}^{b i}$ and $\eta_{k}^{b i}$ are determined with the same procedure used for the tangents. Injecting the Lyapunov-Schmidt reduction in the order $k+1$ system Equation (35a) projected on the left bifurcation mode $\Psi$ and using the condition Equation (35b), the following system is obtained:

$$
\left[\begin{array}{cc}
\left\langle\boldsymbol{\Psi}, \widetilde{\mathrm{Q}}\left(\mathbf{W}, \mathbf{U}_{1}^{b i}\right)\right\rangle & \left\langle\boldsymbol{\Psi}, \widetilde{\mathrm{Q}}\left(\boldsymbol{\Phi}, \mathbf{U}_{1}^{b i}\right)\right\rangle \\
\left\langle\mathbf{W}, \mathbf{U}_{1}^{b i}\right\rangle+\lambda_{1}^{b i} & \left\langle\boldsymbol{\Phi}, \mathbf{U}_{1}^{b i}\right\rangle
\end{array}\right]\left\{\begin{array}{c}
\lambda_{k}^{b i} \\
\eta_{k}^{b i}
\end{array}\right\}=\left\{\begin{array}{c}
-g_{k} \\
-\left\langle\mathbf{V}_{k}^{b i}, \mathbf{U}_{1}^{b i}\right\rangle
\end{array}\right\}
$$

with the coefficient definition

$$
g_{2}=\left\langle\Psi, Q\left(\mathbf{V}_{2}^{b i}, \mathbf{U}_{1}^{b i}\right)\right\rangle
$$

and, for $k>2$,

$$
g_{k}=\left\langle\Psi, Q\left(\mathbf{V}_{k}^{b i}, \mathbf{U}_{1}^{b i}\right)\right\rangle+\sum_{j=2}^{k-1}\left\langle\Psi, \mathbf{Q}\left(\mathbf{U}_{j}^{b i}, \mathbf{U}_{k-j+1}^{b i}\right)\right\rangle .
$$

When a complete power-series is obtained, the range of validity is evaluated. Then, for a chosen tangent, the associated post-bifurcated branches emanating from the bifurcation points are approximated using Equation (16), with $a_{\max }$ or $-a_{\max }$ in 2 directions. This procedure is repeated at wish for each of the computed tangents. Each new starting point on the post-bifurcated branches is either saved for a restart procedure or used directly for new continuation steps.

It should be noted that, in the case of simple steady bifurcation, 4 branches are approximated using 2 tangents and new regular solutions are saved for next restart.

\section{Remark on symmetry-breaking bifurcation case for high-order terms}

For the symmetric post-bifurcated branch, the tangent $\mathbf{X}_{1}^{b s}$ is orthogonal to the bifurcation mode, which leads to the following simplification:

$$
\begin{aligned}
\lambda_{k}^{b s} & =-\frac{\left\langle\mathbf{V}_{k}^{b s}, \mathbf{W}\right\rangle}{\langle\mathbf{W}, \mathbf{W}\rangle+1} \\
\eta_{k}^{b s} & =-\frac{g_{k}^{b s}}{b_{b}} .
\end{aligned}
$$


Then, using the tangent of the antisymmetric branch $\mathbf{X}_{1}^{b a}$, which is exactly the bifurcation mode, the sought coefficients are

$$
\begin{aligned}
\lambda_{k}^{b a} & =-\frac{g_{k}^{b a}}{b_{b}} \\
\eta_{k}^{b a} & =0
\end{aligned}
$$

leading to the following expression of the high-order terms:

$$
\mathbf{U}_{k}^{b a}=\lambda_{k}^{b a} \mathbf{W}+\mathbf{V}_{k}^{b a}
$$

In this particular case, each high-order term of the series is orthogonal to the bifurcation mode according to the pseudo-arc-length condition Equation (35b).

\section{3 | NUMERICAL IMPLEMENTATION}

All the previous numerical methods were implemented in the open-source software ELMER ${ }^{40}$ which is a generic and multiphysics finite element code. Several tests were run with the UMFPACK linear direct solver, which is the default direct solver. For problems requiring memory space greater than $4 \mathrm{~GB}$, the use of an alternative direct solver was mandatory. In this study, the solver MUMPS ${ }^{41}$ was chosen. Accordingly, it was compiled with the OpenBLAS linear algebra library ${ }^{42}$ to get multithreaded operations. Finally, ELMER was compiled to work with MUMPS. All the ANM-based methods and algorithms were implemented in ELMER as new modules and user-defined solvers.*

Additional features are available with the solver MUMPS that makes it possible to compute both Equation (33) and Equation (34) using only one factorization of the augmented tangent operator.

\section{4 | NUMERICAL RESULTS}

Bifurcation analysis is performed for the case of symmetry-breaking steady bifurcation. The critical Reynolds numbers are investigated for the 3D sudden expansion.

The ANM continuation of steady flow solution is performed. Then, the geometric progression criteria Equation (12) and Equation (13) are checked during the continuation. Possibly, steady bifurcations are detected. The critical Reynolds numbers and bifurcation modes are reported. Results obtained with the ANM methods in ELMER are compared with the bifurcation information of the literature.

Computations presented in this paper were performed on a DELL Precision T7500 using 2 Intel(R) Xeon(R) X5677 @ $3.47 \mathrm{GHz}$ (16 threads) with $96 \mathrm{~GB}$ of memory or on a Dell PowerEdge R930 using 2 Intel(R) Xeon(R) CPU E7-8860 v4 @ $2.20 \mathrm{GHz}$ (72 threads) with $1 \mathrm{~TB}$ of memory.

This section is organized as follows. Firstly, the 3D sudden expansion case is described. Secondly, the first primary bifurcation is described for several geometric ratios. Then, the other primary bifurcations are investigated. Finally, secondary bifurcations are presented for one geometric ratio.

\section{1 | Test case description}

The 3D sudden expansion test case is well documented in other works. ${ }^{22-25}$ Bifurcation analysis using the aspect ratio $E=3$ was proposed numerically in other works ${ }^{26-29}$ and, more recently, in the work of Medale and Cochelin ${ }^{14}$ using ANM-based methods. The fluid domain and the boundary conditions are reported in Figure 1. The length $L$ is chosen long enough to capture the steady bifurcation phenomenon. Mesh features with several geometric ratios $A_{i}=W / h$ and length $L$ are presented in Table 1.

Linear interpolation $\mathrm{H} 8 / 8 \mathrm{C}$ stabilized finite elements are used. ${ }^{43}$

${ }^{*}$ https://github.com/YGuevel/ElmerMAN 


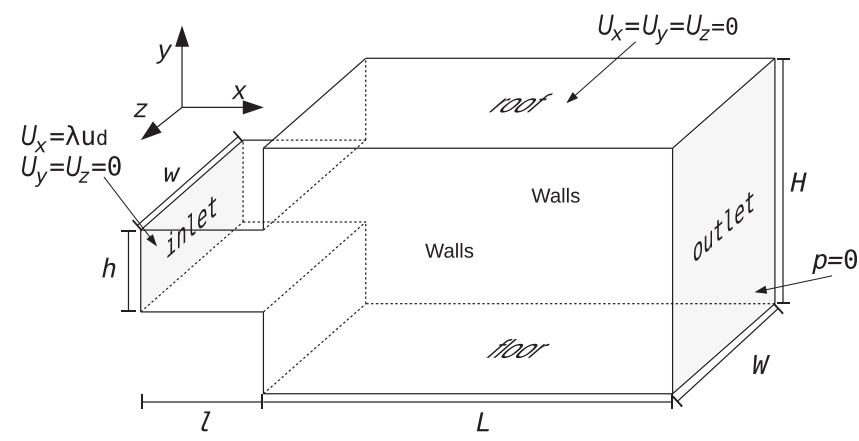

FIGURE 1 Sudden expansion. $h=10, H=3 h, l=3 h, L, W=A_{i} h$

TABLE 1 Meshes for the case $E=3, l=3 h$, and ratios $A_{i}=W / h$. Length $L$, number of H8 elements for entrance $(\mathrm{Nh} \times \mathrm{NW} \times \mathrm{Nl})$ and body $(\mathrm{NH} \times \mathrm{NW} \times \mathrm{NL})$, half-bandwidth $(\mathrm{HBW})$, memory space, and CPU factorization time required by MUMPS are given. (v4) stands for mono-threaded computation using the MUMPS V4 library, otherwise the MUMPS V5 and OpenBlas libraries are used

\begin{tabular}{|c|c|c|c|c|c|c|c|c|c|}
\hline \multirow[b]{2}{*}{$A_{i}$} & \multirow[b]{2}{*}{$L$} & \multicolumn{2}{|c|}{ Number of $\mathrm{H} 8$ Elements } & \multirow{2}{*}{$\begin{array}{r}\text { Number of } \\
\text { Nodes }\end{array}$} & \multirow{2}{*}{$\begin{array}{r}\text { Number of } \\
\text { DOF }\end{array}$} & \multirow[b]{2}{*}{ HBW } & \multirow[b]{2}{*}{ RAM } & \multirow[b]{2}{*}{ LU } & \multirow[b]{2}{*}{ Thread } \\
\hline & & Entrance & Body & & & & & & \\
\hline 1 & $30 \mathrm{~h}$ & $12 \times 12 \times 36$ & $36 \times 12 \times 360$ & 179725 & 718900 & 1131 & $14 \mathrm{~GB}$ & $330 \mathrm{~s}$ & $8 \mathrm{~T}$ \\
\hline 1 & $60 \mathrm{~h}$ & $22 \times 22 \times 66$ & $66 \times 22 \times 1320$ & 2070575 & 8282300 & 3611 & $368 \mathrm{~GB}$ & $1550 \mathrm{~s}$ & $36 \mathrm{~T}$ \\
\hline 1.25 & $30 \mathrm{~h}$ & $16 \times 20 \times 48$ & $48 \times 20 \times 480$ & 512085 & 2048340 & 2415 & $75 \mathrm{~GB}$ & $940 \mathrm{~s}$ & $8 \mathrm{~T}$ \\
\hline 1.5 & $60 \mathrm{~h}$ & $12 \times 18 \times 36$ & $36 \times 18 \times 720$ & 515755 & 2063020 & 1653 & $58 \mathrm{~GB}$ & $1800 \mathrm{~s}$ & $1 \mathrm{~T}(\mathrm{v} 4)$ \\
\hline 2 & $30 \mathrm{~h}$ & $12 \times 24 \times 36$ & $36 \times 24 \times 360$ & 345625 & 1382500 & 2534 & $45 \mathrm{~GB}$ & $1800 \mathrm{~s}$ & $1 \mathrm{~T}(\mathrm{v} 4)$ \\
\hline 2 & $50 \mathrm{~h}$ & $16 \times 32 \times 48$ & $48 \times 32 \times 800$ & 1322145 & 5288580 & 4466 & $256 \mathrm{~GB}$ & $1100 \mathrm{~s}$ & $36 \mathrm{~T}$ \\
\hline 2.5 & $30 \mathrm{~h}$ & $12 \times 30 \times 36$ & $36 \times 30 \times 360$ & 428575 & 1714300 & 3416 & $68 \mathrm{~GB}$ & $1000 \mathrm{~s}$ & $8 \mathrm{~T}$ \\
\hline 3 & $30 \mathrm{~h}$ & $8 \times 24 \times 24$ & $24 \times 24 \times 240$ & 156025 & 624100 & 2162 & $17 \mathrm{~GB}$ & $550 \mathrm{~s}$ & $1 \mathrm{~T}(\mathrm{v} 4)$ \\
\hline 3 & $50 \mathrm{~h}$ & $14 \times 42 \times 42$ & $42 \times 42 \times 700$ & 1323239 & 5292956 & 6470 & 235 GB & $1300 \mathrm{~s}$ & $36 \mathrm{~T}$ \\
\hline 3.5 & $30 \mathrm{~h}$ & $8 \times 28 \times 24$ & $24 \times 28 \times 240$ & 180989 & 723956 & 2220 & $22 \mathrm{~GB}$ & $860 \mathrm{~s}$ & $1 \mathrm{~T}(\mathrm{v} 4)$ \\
\hline 3.75 & $30 \mathrm{~h}$ & $10 \times 37 \times 30$ & $30 \times 37 \times 300$ & 367118 & 1468472 & 3414 & $59 \mathrm{~GB}$ & $855 \mathrm{~s}$ & $8 \mathrm{~T}$ \\
\hline 4 & $30 \mathrm{~h}$ & $10 \times 40 \times 30$ & $30 \times 40 \times 300$ & 396101 & 1584404 & 3414 & $66 \mathrm{~GB}$ & $1000 \mathrm{~s}$ & $8 \mathrm{~T}$ \\
\hline 4 & $40 \mathrm{~h}$ & $14 \times 56 \times 42$ & $42 \times 56 \times 560$ & 1757463 & 5643684 & 6570 & $340 \mathrm{~GB}$ & $1700 \mathrm{~s}$ & $36 \mathrm{~T}$ \\
\hline 5 & $30 \mathrm{~h}$ & $10 \times 50 \times 30$ & $30 \times 50 \times 300$ & 492711 & 1970844 & 3493 & $91 \mathrm{~GB}$ & $1500 \mathrm{~s}$ & $8 \mathrm{~T}$ \\
\hline 5 & $40 \mathrm{~h}$ & $14 \times 70 \times 42$ & $42 \times 70 \times 560$ & 1757463 & 7029852 & 6737 & $476 \mathrm{~GB}$ & $2900 \mathrm{~s}$ & $36 \mathrm{~T}$ \\
\hline 6 & $30 \mathrm{~h}$ & $8 \times 48 \times 24$ & $24 \times 48 \times 240$ & 305809 & 1223236 & 2667 & $48 \mathrm{~GB}$ & $3000 \mathrm{~s}$ & $1 \mathrm{~T}(\mathrm{v} 4)$ \\
\hline 6 & $30 \mathrm{~h}$ & $15 \times 90 \times 45$ & $45 \times 90 \times 450$ & 1953406 & 7813624 & 9093 & 604 GB & $3600 \mathrm{~s}$ & $36 \mathrm{~T}$ \\
\hline 7 & $30 \mathrm{~h}$ & $9 \times 63 \times 27$ & $27 \times 63 \times 270$ & 502912 & 2011648 & 3855 & 96 GB & $1800 \mathrm{~s}$ & $8 \mathrm{~T}$ \\
\hline 7 & $30 \mathrm{~h}$ & $15 \times 105 \times 45$ & $45 \times 105 \times 450$ & 2275396 & 9101584 & 10473 & $749 \mathrm{~GB}$ & $4900 \mathrm{~s}$ & $48 \mathrm{~T}$ \\
\hline 8 & $30 \mathrm{~h}$ & $8 \times 70 \times 24$ & $24 \times 70 \times 240$ & 486989 & 1947956 & 3785 & $89 \mathrm{~GB}$ & $1500 \mathrm{~s}$ & $8 \mathrm{~T}$ \\
\hline 8 & $55 \mathrm{~h}$ & $12 \times 96 \times 36$ & $36 \times 96 \times 714$ & 2417725 & 9670900 & 7647 & $563 \mathrm{~GB}$ & $4300 \mathrm{~s}$ & $48 \mathrm{~T}$ \\
\hline 9 & $30 \mathrm{~h}$ & $8 \times 72 \times 24$ & $24 \times 72 \times 240$ & 455593 & 1822372 & 3867 & 83 GB & $1500 \mathrm{~s}$ & $8 \mathrm{~T}$ \\
\hline 9 & $40 \mathrm{~h}$ & $12 \times 108 \times 36$ & $36 \times 108 \times 360$ & 1990885 & 3981770 & 8535 & $592 \mathrm{~GB}$ & $3300 \mathrm{~s}$ & $36 \mathrm{~T}$ \\
\hline 10 & $30 \mathrm{~h}$ & $8 \times 80 \times 24$ & $24 \times 80 \times 240$ & 505521 & 2022084 & 4267 & $96 \mathrm{~GB}$ & $1800 \mathrm{~s}$ & $8 \mathrm{~T}$ \\
\hline 10 & $40 \mathrm{~h}$ & $12 \times 120 \times 36$ & $36 \times 120 \times 480$ & 2210065 & 8840260 & 9423 & $662 \mathrm{~GB}$ & $4300 \mathrm{~s}$ & $36 \mathrm{~T}$ \\
\hline
\end{tabular}

An established velocity profile is prescribed on the inlet. Many formulations can be found in the literature. The best-fit profile compared to a gravitational driven flow has been used in this study.

Firstly, Shah and London ${ }^{44(\text { eq. } 332)}$ and Titarev and Shakhov ${ }^{45(\text { eq. 3.3) }}$ proposed similar analytical formulation based on infinite sums of hyperbolic terms. Secondly, in the works of Baloch et al, ${ }^{24(\text { eq. } 18)}$ Theofilis et al, ${ }^{46(\text { eq. } 2.3)}$ and Nicolas et al, ${ }^{47(e q .7)}$ another formulation is obtained. Moreover, in the work of Spiga and Morino, ${ }^{48(e q .7)}$ only trigonometric functions are used.

Finally, the analytic formulation used in this work is based on the work of Tanyeri et al. ${ }^{49(\mathrm{~S} 1)}$ It uses infinite sum of hyperbolic terms in $z$ for the spanwise direction multiplied with a classic parabolic profile for $y$, the height. It has been 
decided to modulate this latter parabolic profile as proposed in the work of Shah and London ${ }^{44(e q s .334-337)}$ using a power exponent $n$. The inlet velocity profile that best fits a gravitational driven flow using ELMER is

$$
\mathbf{u}_{d x}(y, z)=C\left(1-\left(\frac{2 y}{h}\right)^{2}\right)^{n} \sum_{k=1,3, . .}^{\infty} \frac{1}{k^{3}}\left(1-\frac{\cosh \left(\frac{k \pi z}{h}\right)}{\cosh \left(\frac{k \pi W}{2 h}\right)}\right),
$$

where $C$ is a normalization coefficient. Several tests were performed such that the values $k \in[1,15]$ and $n=0.95$ have been used in this work.

\section{2 | Primary bifurcation}

The ANM continuation is performed to follow the steady solution branches. The ANM parameters are $N=30$ and $\eta=$ $10^{-16}$. Steady bifurcation detection is performed with $\epsilon_{g p 1}=10^{-6}$ and $\epsilon_{g p 2}=10^{-3}$. Using these parameters, the ANM method requires from 10 to 14 steps to detect the first bifurcation. Other parameters, for example, $\eta=10^{-8}$, allow to detect accurately the first primary critical values with less steps (around 5 to $7 \mathrm{step}$ ). However, it leads to degraded quality of solutions.

Some computational cost information are given. The CPU factorization time presented in Table 1 is highly decreased because a multithreaded library has been used. Usually, in ANM, the tangent operator factorization represents the main part of the computational time. In this work, when using fine meshes with long bodies, it requires 3 "multithreaded LU" CPU time to perform one ANM step. The geometric progression detection is based on vector manipulations, which is negligible. Then, some specific vectors need to be computed. Due to MUMPS V5, it is possible to factorize only one augmented system to get all the specific vectors and to compute the post-bifurcated branches. Finally, the branch-switching technique allows to compute 4 parts of the post-bifurcated branches using only 1.66 ANM step. This represents half of a working day for standard meshes to a day or two for meshes with almost $10^{7}$ degrees of freedom using 36 threads for the factorization step.

Readers may get further details of the ANM computational cost from other works. ${ }^{12-15,17-20}$

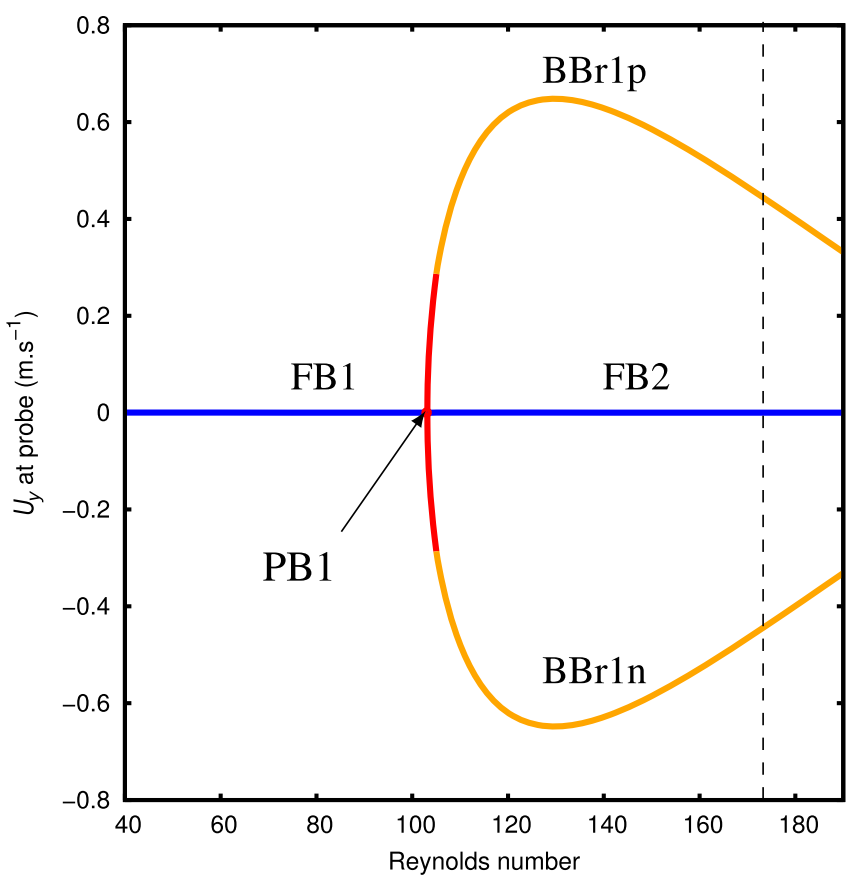

FIGURE 2 Bifurcation diagram for $A_{i}=8$. FB1 and FB2 denote fundamental branches, PB1 denotes the first primary bifurcation point, and BBr1n and BBr1p denote the post-critical unsymmetric nonlinear branches. Blue: fully symmetric solutions. Red: one ANM step using the singular initial solution. Orange: bifurcated branches with a broken symmetry but still not unsymmetrical 


\subsection{1 । Bifurcation diagram}

A velocity probe is used at coordinates $(100,0,0)$ at the intersection of the vertical and horizontal symmetry planes. A 2D bifurcation diagram may be plotted using either $\mathbf{u}_{y}$ or $\mathbf{u}_{z}$ versus the Reynolds number. This choice depends on which symmetry breaking is investigated.

A bifurcation diagram is proposed for the first primary bifurcation in Figure 2. From the fundamental branch FB1, a pitchfork bifurcation is detected. Then, the singular solution $\mathbf{U}_{c}$ and the 3 specific vectors required by the branch-switching technique are computed. The red curve depicted one ANM step starting with a singular solution. A new regular point is chosen to follow an other branch.

\subsection{2 | Flow topology descriptions}

Illustration of the 3D flow is depicted in Figure 3. Two Reynolds numbers are considered, ie, the critical value $\operatorname{Re}_{c}=103$ and a higher value $\mathrm{Re}=174$ for which 3 solutions exist (see dashed line in Figure 2). The streamlines of the flow solutions allow us to identify recirculation zones and vortex lines.

The first primary bifurcation breaks the horizontal plane, and the vertical symmetry plane being still present.

In order to validate the flow topology, features are determined using skin friction lines. ${ }^{50}$ It is depicted in skin friction lines on the floor, roof, and lateral wall in Figure 4 for the flow solution on the BBr1n branch. The kind of singularities and their positions are in good agreement with the pattern depicted in the work of Chiang et al. ${ }^{27}$

\subsection{3 । Evolution of $\operatorname{Re}_{c} \operatorname{vs} A_{i}$}

Critical Reynolds numbers for the first primary bifurcation are reported in Table 2 for several geometric aspect ratios $A_{i}$. Values from the literature are also reported. Moreover, a transition from a symmetrical state for $A_{i}=1.5$ at $\operatorname{Re}=314$ to an unsymmetrical one for $A_{i}=2$ at $\mathrm{Re}=297$ was presented in the work of Tsui and Wang. ${ }^{29}$ In the work of Chiang et al, ${ }^{27}$

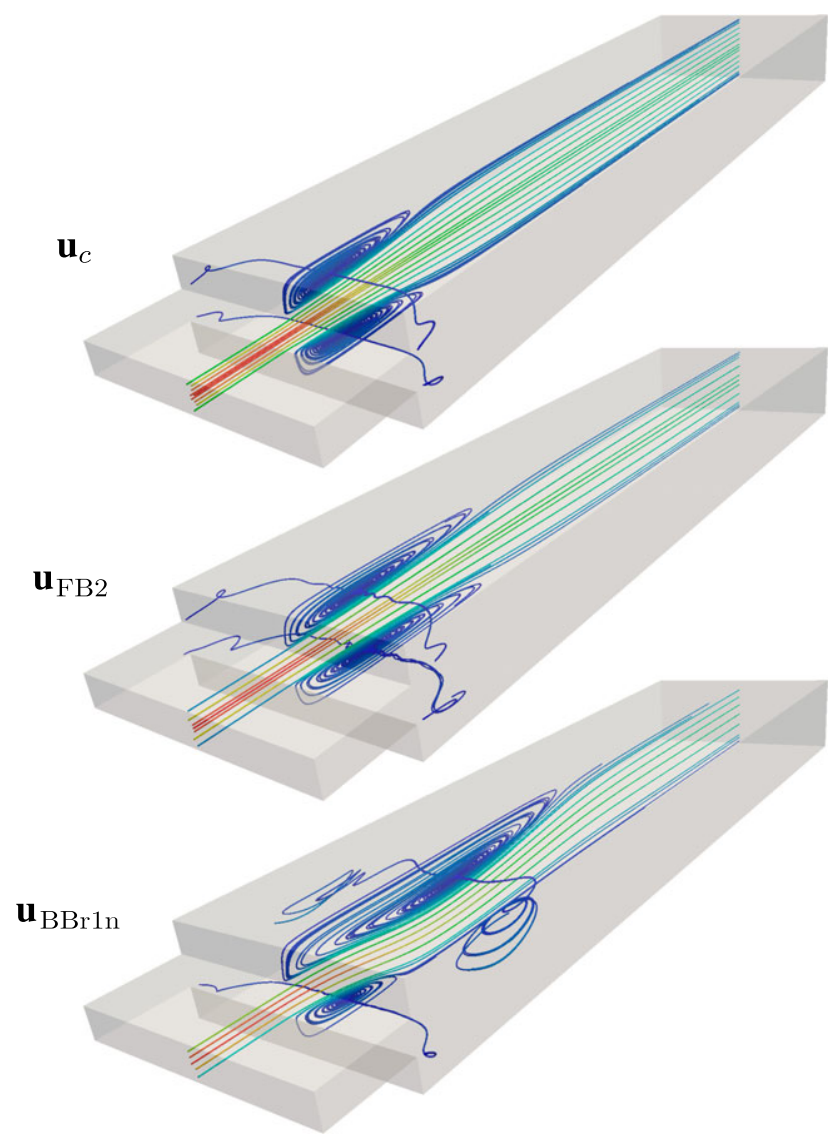

FIGURE 3 Streamlines for 3-dimensional steady flows, $A_{i}=8, L=30 h . \mathbf{u}_{c}$ at $\operatorname{Re}_{c}=103$, $\mathbf{u}_{F B 2}$ and $\mathbf{u}_{\mathrm{BBr} 1 \mathrm{n}}$ are both evaluated at Re $=174$ [Colour figure can be viewed at wileyonlinelibrary.com] 

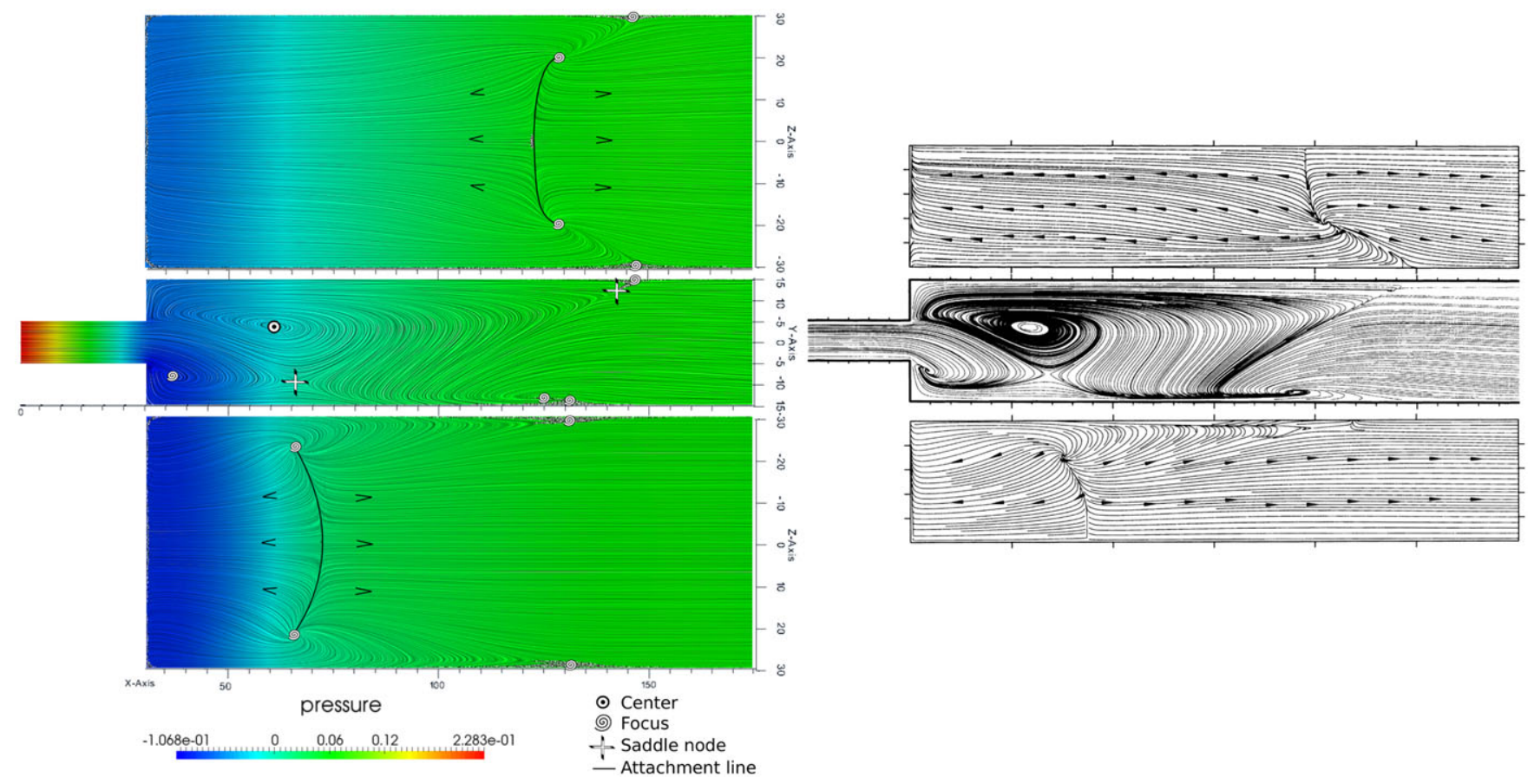

FIGURE 4 Skin friction for the postcritical BBr1n branch of the sudden expansion with $A_{i}=6$ at Re $=135$. (Left) our study, made with ParaView with line integral convolution; (Right) extracted from the work of Chiang et al, ${ }^{27}$ half-domain for roof and floor plane [Colour figure can be viewed at wileyonlinelibrary.com]

TABLE 2 Critical Reynolds number associated to the first primary steady bifurcation for several $A_{i}$. "FL" stands for finer mesh or longer body, "NS" stands for not searched

\begin{tabular}{|c|c|c|c|c|}
\hline \multirow[b]{2}{*}{$A_{i}=W / h$} & \multicolumn{2}{|c|}{ This Study } & \multirow[b]{2}{*}{ Literature } & \multirow[b]{2}{*}{$\mathbf{R e}_{c}$} \\
\hline & $\mathbf{R e}_{c \mathrm{~PB} 1}$ & $\operatorname{Re}_{c P B 1}(F L)$ & & \\
\hline 1 & $(>450)$ & 734.7 & Tsui and Wang ${ }^{29}$ & $>300$ \\
\hline 1.25 & 438.7 & NS & & \\
\hline 1.5 & 333.3 & NS & & \\
\hline 2 & 241.7 & 241.7 & & \\
\hline 2.5 & 196.1 & NS & & \\
\hline 3 & 170.6 & 170.2 & Tsui and Wang ${ }^{29}$ & 171 \\
\hline 3.5 & 149.9 & NS & Chiang et $\mathrm{al}^{27}$ & 145 \\
\hline 3.75 & 142.5 & NS & & \\
\hline 4 & 136.7 & 136.2 & & \\
\hline 5 & 121.2 & 120.7 & & \\
\hline 6 & 112.7 & 111.5 & Schreck and Schäfer ${ }^{26}$ & 113 \\
\hline 7 & 106.9 & 106.0 & & \\
\hline 8 & 103.1 & 102.4 & & \\
\hline 9 & 100.5 & 99.4 & & \\
\hline 10 & 98.3 & 97.3 & Medale and Cochelin ${ }^{14}$ & 98.3 \\
\hline
\end{tabular}

a transition was noted from a symmetrical state for $A_{i}=3.5$ at $\operatorname{Re}=145$ to unsymmetrical one for $A_{i}=3.75$ at $\operatorname{Re}=143$. Those transitions are reported respectively in orange and red in Figure 5.

Additional information is given regarding critical Reynolds numbers found in the literature. In the work of Tsui and Wang, ${ }^{29}$ inlet flow is uniform and mean velocity is used for the Reynolds number definition, so that coefficients are applied to match our definition. In Table 3, some ratios $u_{\max } / \frac{3}{2} u_{\text {mean }}$ are given. Coefficient for aspect ratios $A_{i}=1.5$ and $A_{i}=2$ are numerically evaluated using simulation of a flow in a rectangular duct. For higher aspect ratios, values are extracted from table 4 in the work of Chiang et al. ${ }^{27}$ 


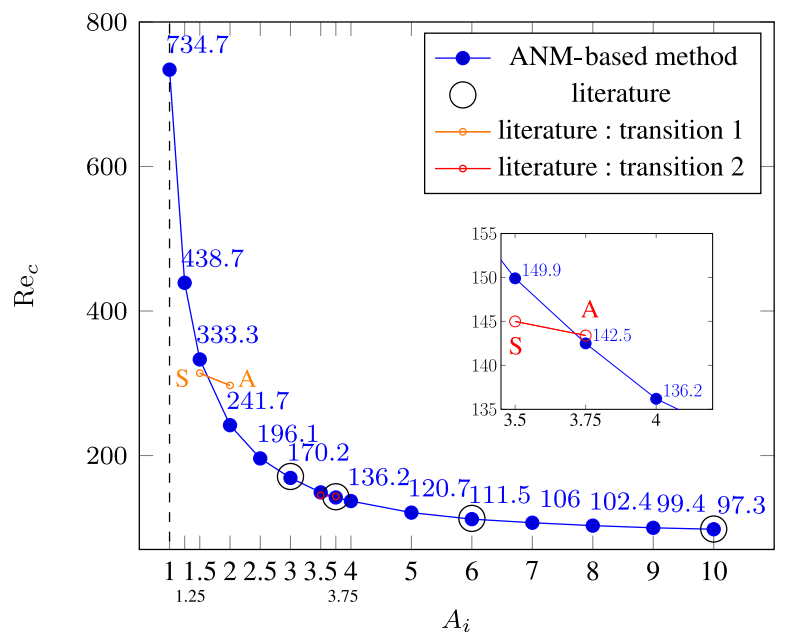

FIGURE 5 Critical Reynolds number associated to the first primary steady bifurcation for several $A_{i}$ using the asymptotic-numerical method (ANM)-based method. In black circles, the value from literature. In red and orange, the transition from symmetric flow (S) to antisymmetric flow (A) found in the literature

TABLE 3 Correspondence between $u_{\max }$ and $\frac{3}{2} u_{\text {mean }}$ for a uniform velocity inlet in a rectangular duct. Values higher than $A_{i}=3$ are extracted from table 4 in the work of Chiang et $\mathrm{al}^{27}$

\begin{tabular}{lc}
$\boldsymbol{A}_{\boldsymbol{i}}$ & Ratio $\boldsymbol{U}_{\max } / \frac{3}{2} \boldsymbol{U}_{\text {mean }}$ \\
\hline 1.5 & 1.576 \\
2 & 1.488 \\
3 & 1.242 \\
3.5 & 1.209 \\
3.75 & 1.195 \\
6 & 1.117 \\
\hline
\end{tabular}

Firstly, it can be observed that our results are in very good agreement with the literature. Secondly, between standard mesh and finer and/or longer mesh (see Table 1), critical values are very similar. This indicates that standard mesh with $L=30$ is sufficient to accurately compute critical values for the first primary bifurcation.

To the best knowledge of the authors, no result was found in the literature for the ratio $A_{i}=1$. In the work of Tsui and Wang, ${ }^{29}$ no primary bifurcation was found for Reynolds number lower than $\mathrm{Re}=300$. Using standard mesh, we were not able to compute accurately fundamental solutions for Reynolds number higher than $\operatorname{Re}=450$. However, with a finer and longer mesh, the first primary bifurcation was detected at $\operatorname{Re}_{c \mathrm{~PB} 1}=734.7$.

These values are plotted in Figure 5. The trend for high values of aspect ratio is to converge slowly toward the critical Reynolds number $\mathrm{Re}_{c}=80.4$ obtained for the $2 \mathrm{D}$ flow. ${ }^{15}$ This is in perfect agreement with critical values for higher ratios computed in the work of Medale and Cochelin. ${ }^{14}$ Finally, it is observed that the first primary bifurcation occurs for higher Reynolds number as the aspect ratio is decreasing.

\subsubsection{B Bifurcation vectors description}

A spatial representation of the bifurcation modes $\boldsymbol{\Phi}$ and $\boldsymbol{\Psi}$ and of the particular solution $\mathbf{W}$ is depicted in Figure 6 . In the same way, the pressure and the velocity parts of these 2 bifurcation modes are depicted in Figures 7 and 8, respectively.

The velocity part of the bifurcation mode $\boldsymbol{\Phi}$ is in agreement with the work of Medale and Cochelin. ${ }^{14}$ Moreover, the vertical middle cut plane for the pressure part is similar to the $2 \mathrm{D}$ case. ${ }^{51}$

It is observed that the left bifurcation mode $\boldsymbol{\Psi}$ drives the inlet flow. This behavior is in agreement with the work of Battaglia et al. ${ }^{51}$ 


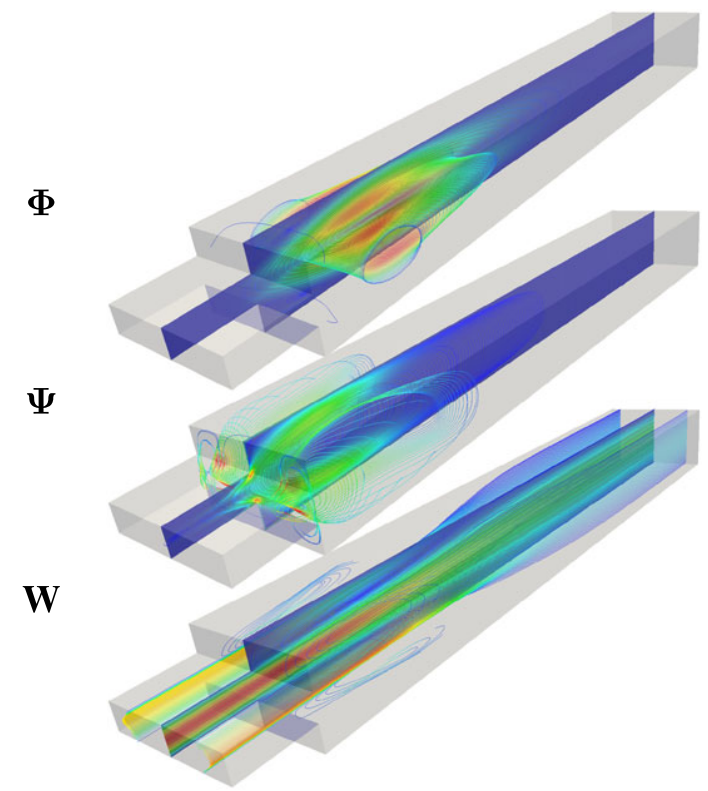

FIGURE 6 Slice and streamlines for the singular tangent operator specific vectors at first steady primary bifurcation (PB1). Bifurcation mode $\boldsymbol{\Phi}$, left bifurcation mode $\boldsymbol{\Psi}$, and specific solution $\mathbf{W}$. Case of $A_{i}=5$
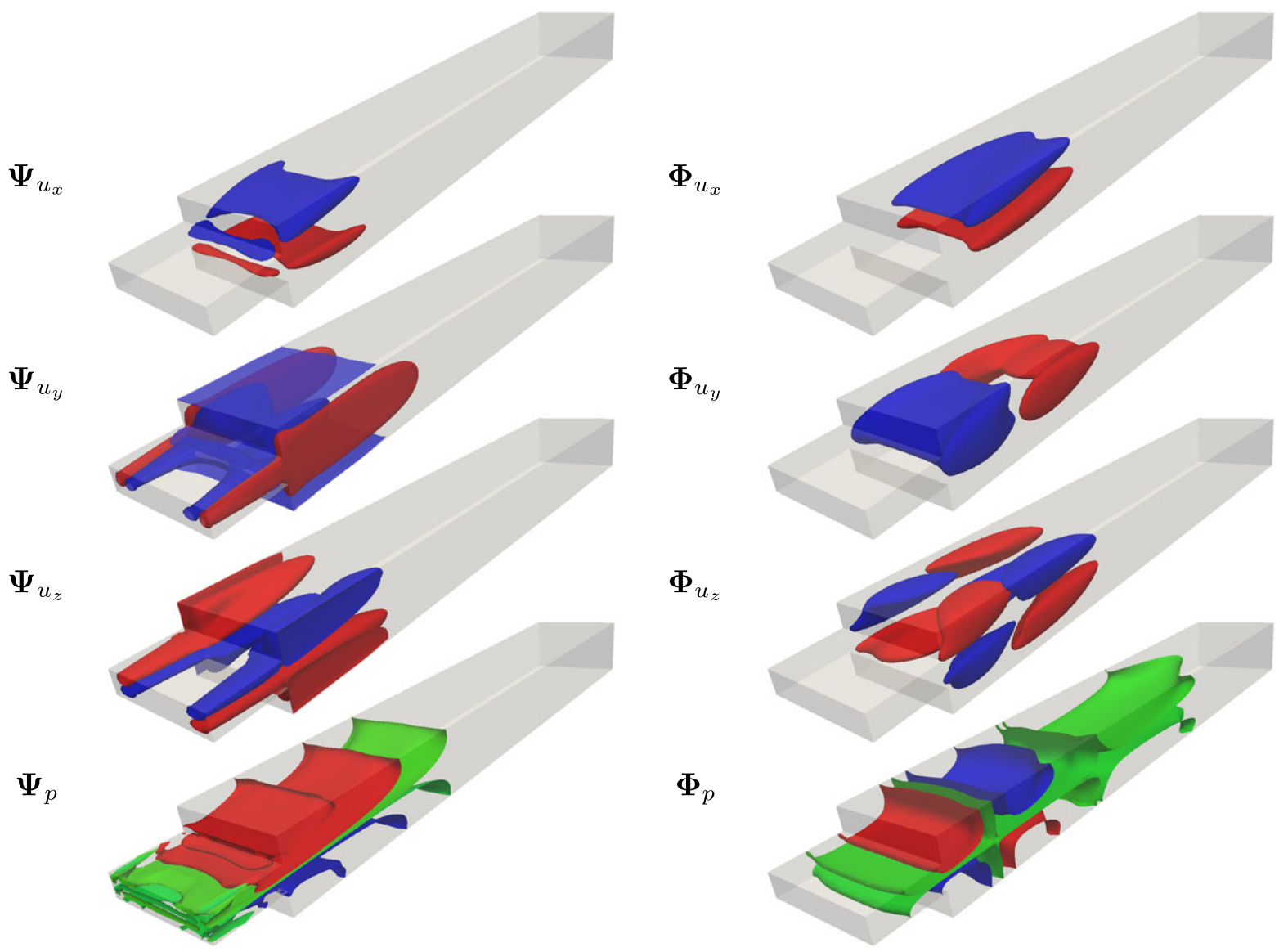

FIGURE 7 Left bifurcation mode $\boldsymbol{\Psi}_{\mathrm{PB} 1}$ (Left) and bifurcation mode $\boldsymbol{\Phi}_{\mathrm{PB} 1}$ (right). Velocity components and pressure for $E=3 A_{i}=5 L=30$. Contours for $\boldsymbol{\Psi}_{u}$ are $10^{-2}, 2 \cdot 10^{-3}, 2 \cdot 10^{-3}$. Contours for $\boldsymbol{\Phi}_{u}$ are $10^{-3}, 5 \cdot 10^{-4}, 10^{-4}$. Red: positive; blue: negative; green: $10^{-6}$ 


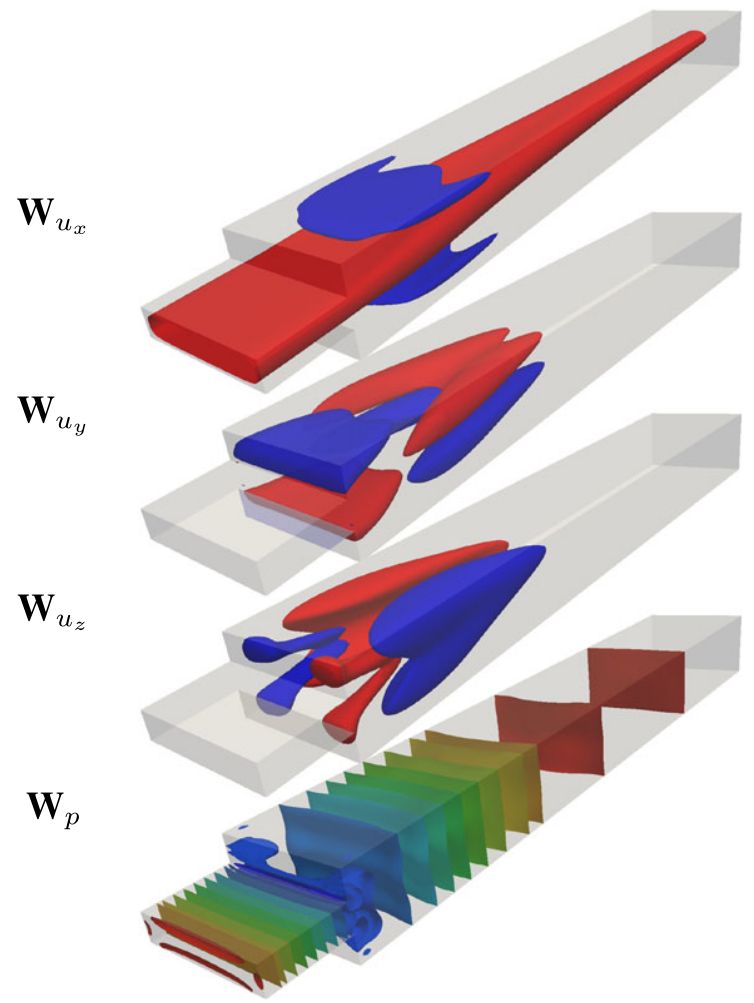

FIGURE 8 Particular solution $\mathbf{W}_{\mathrm{PB} 1}$. Velocity components and pressure for $E=3 A_{i}=5 L=30$. Contours for $\mathbf{W}_{u}$ are $5 \cdot 10^{-4}, 7 \cdot 10^{-5}$, and $5 \cdot 10^{-5}$. Red: positive; blue: negative

TABLE 4 Critical Reynolds numbers and bifurcation type of second and third primary bifurcation for several geometric ratios. "Std" stands for standard mesh, "F/L" for finer or longer mesh, "NS" for not searched.

\begin{tabular}{|c|c|c|c|c|c|c|c|c|}
\hline \multirow[b]{2}{*}{$\boldsymbol{A}_{\boldsymbol{i}}=\boldsymbol{W} / \boldsymbol{h}$} & \multicolumn{4}{|c|}{$\mathbf{R e}_{c \mathrm{~PB} 2}$} & \multicolumn{4}{|c|}{$\mathbf{R e}_{c \mathrm{~PB} 3}$} \\
\hline & Std & type & $\mathbf{F} / \mathbf{L}$ & type & Std & type & F/L & type \\
\hline 2 & NS & & 778.6 & $\Delta$ & NS & & NS & \\
\hline 3 & NS & & 541.5 & $\Delta$ & NS & & 881.1 & $\vartheta$ \\
\hline 4 & NS & & 427.9 & $\Delta$ & NS & & 734.7 & $\vartheta$ \\
\hline 5 & NS & & 372.7 & $\Delta$ & NS & & 664.1 & $\vartheta$ \\
\hline 6 & 337.8 & $\Delta$ & 338.2 & $\Delta$ & NS & & 614.4 & $\vartheta$ \\
\hline 7 & 313.3 & $\Delta$ & 308.3 & $\square$ & 313.8 & $\square$ & 314.8 & $\Delta$ \\
\hline 8 & 204.7 & $\square$ & 203.9 & $\square$ & 296.4 & $\Delta$ & 294.6 & $\Delta$ \\
\hline 9 & 161.3 & $\square$ & 159.2 & $\square$ & 283.5 & $\Delta$ & 283.5 & $\Delta$ \\
\hline 10 & 138.2 & $\square$ & 136.9 & $\square$ & 273.6 & $\Delta$ & 274.4 & $\Delta$ \\
\hline
\end{tabular}

\subsection{5 | Evolution of additional primary bifurcations}

Beyond the first primary bifurcation, fundamental branches are computed for various geometric ratios. Then, additional primary bifurcations are found. For each primary bifurcation, it was observed that bifurcation mode topologies were very closed to each others. Moreover, it is reported in Tables 4 and 5 the values of the critical Reynolds number for the primary bifurcation with respect to the aspect ratio $A_{i}$. Accordingly, it was proposed to track the evolution of the critical Reynolds numbers for each primary bifurcation with respect to the aspect ratio $A_{i}$ (see Figure 10). Whatever the rank of the primary bifurcation, it was observed that the greater the aspect ratio is, the lower the critical Reynolds numbers are. The different kinds of bifurcation modes for the primary bifurcation noted as $\boldsymbol{\Lambda}, \square$, and $\boldsymbol{\nabla}$ are depicted in Figures 11 and 12 for the case of $A_{i}=8$. 
TABLE 5 Critical Reynolds numbers and bifurcation type for the fourth and fifth primary bifurcations versus the aspect ratio $A_{i}$. "Std" stands for standard mesh, "F/L" for finer or longer mesh, "NS" for not searched, and "NF" for not found

\begin{tabular}{|c|c|c|c|c|c|c|c|c|}
\hline \multirow[b]{2}{*}{$A_{i}=W / h$} & \multicolumn{4}{|c|}{$\mathbf{R e}_{c \mathrm{~PB} 4}$} & \multicolumn{4}{|c|}{$\mathbf{R e}_{c \mathrm{~PB} 5}$} \\
\hline & Std & type & $\mathbf{F} / \mathbf{L}$ & type & Std & type & F/L & type \\
\hline 8 & 557.5 & $\vartheta$ & 568.8 & 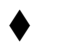 & NS & & NS & \\
\hline 9 & $\mathrm{NF}$ & & 545.0 (Padé) & & NS & & NS & \\
\hline 10 & 441.0 & $\oplus$ & 432.9 & $\oplus$ & 518.7 & 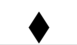 & 526.6 & 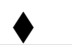 \\
\hline
\end{tabular}
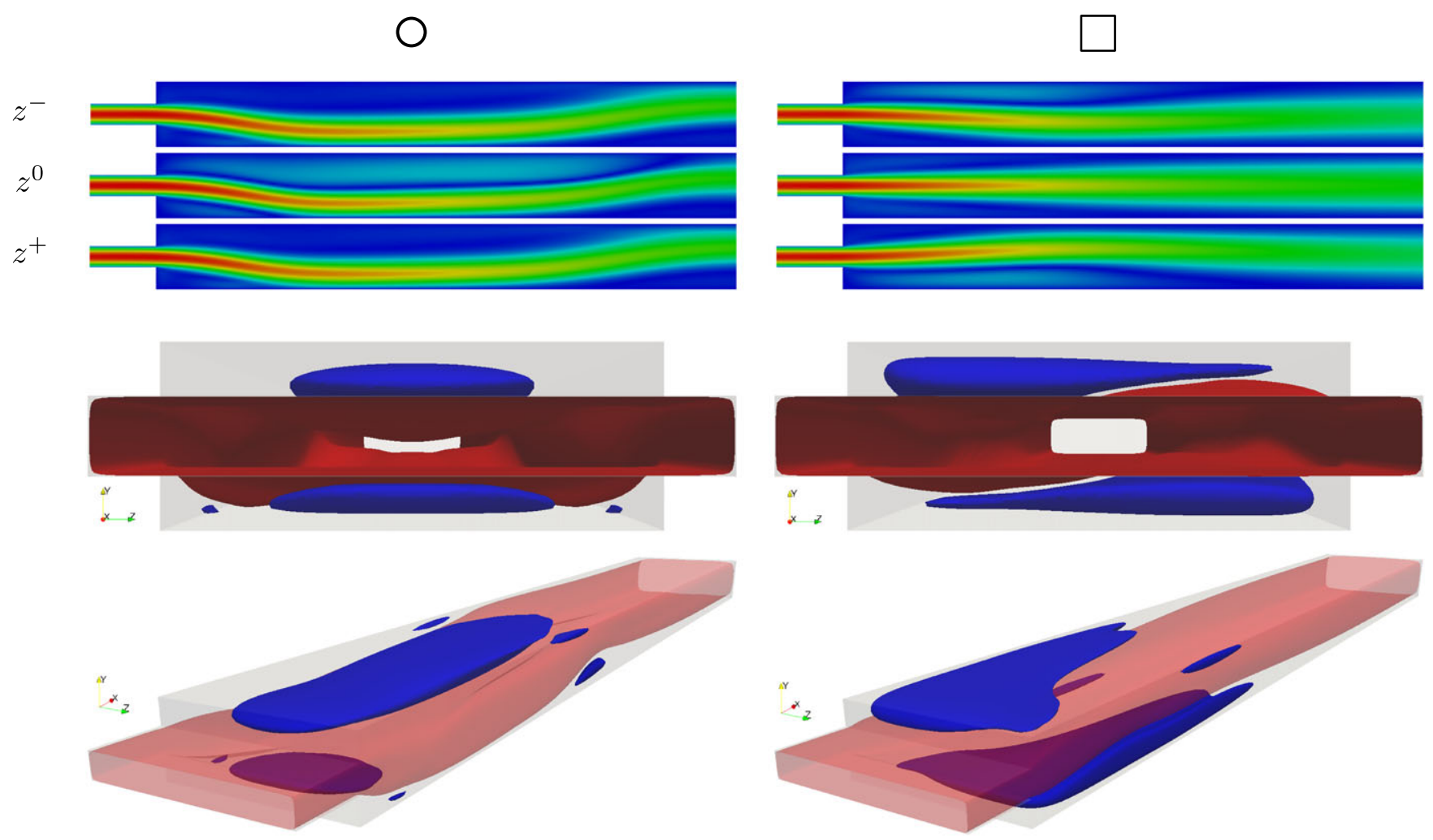

FIGURE 9 Velocity comparison of flows on the post-bifurcated branches BBr1n (post $\circ$ at $\operatorname{Re}=444$ ) and BBr2n (post $\square$ at $\operatorname{Re}=315$ ) for the case $E=3, A_{i}=8, L=55 h$. Up: crop of 3 planar slices, at $z^{-}=-25, z^{0}=0$, and $z^{+}=25$, of velocity magnitude at different spanwise coordinates. Middle: front view of the $\mathbf{u}_{x}$ velocity component signed contours: 0.2 (red) and -0.2 (blue). Down: overview of those contours with transparency for the positive value

In Table 4, the critical Reynolds numbers are similar for standard and finer or longer meshes. Some bifurcations were not searched using standard meshes. The recirculation zones were not correctly captured with $L=30$ because it was necessary to refine the mesh for higher Reynolds number. However, the recirculation zones were correctly computed using finer or longer meshes.

For the ratio $A_{i}=10$, critical values issued from the work of Medale and Cochelin ${ }^{14}$ are $\operatorname{Re}_{c \mathrm{~PB} 2}=137.60, \operatorname{Re}_{c \mathrm{~PB} 3}=210.86$, and $\operatorname{Re}_{c \mathrm{~PB} 4}=280.05$. Firstly, only two of these 3 additional bifurcations are detected. Even with a Newton corrector using a residual norm criterion prescribed at $10^{-6}$ and a finer mesh, we were not able to detect the bifurcation at $\operatorname{Re}_{c}=210.86$. However, the critical values and kinds of bifurcation are in agreement with the literature.

The primary bifurcation, noted as $\square$, breaks both planar symmetries. The spanwise symmetry is broken. The flow is in the symmetry group $\mathbf{Z}_{2}(y) \oplus \mathbf{Z}_{2}(z)$ as described in the work of Chiang et al. ${ }^{28}$ More precisely, this is an X-axis symmetry. In Figure 9, a visual comparison between flows on post-bifurcated branches BBr1 (post o) and BBr2 (post $\square$ ) is proposed. Firstly, in the case of the spanwise bifurcation, planes $z=-25$ and $z=25$ are antisymmetric. Moreover, slice at middle 
plane shows a symmetric pattern. Whereas, the topology of the BBr1 flow for the 3 slices is in the same top-bottom direction. Secondly, contours make it possible to see differences between the BBr1 branch flow, which breaks the top-bottom symmetry and is in the planar symmetry group $\mathbf{Z}_{2}(z)$, and the $\mathrm{BBr} 2$ branch flow, which breaks both plane of symmetry and is in the $\mathbf{Z}_{2}(y) \oplus \mathbf{Z}_{2}(z)$ group. The latter flow is clearly symmetric according to the $\mathrm{X}$-axis. This spanwise symmetry breaking was not found below $A_{i}=30$ in the work of Chiang et al ${ }^{28}$ In the work of Medale and Cochelin,,${ }^{14}$ this bifurcation was still present for ratios $A_{i} \geq 10$. In the present study, this spanwise bifurcation persists from $A_{i}=7$ to $A_{i}=10$. It is not found for smaller ratios. Another interesting behavior with $A_{i}=7$, is the permutation in the order of primary bifurcation. Using standard mesh, the spanwise bifurcation is found at $\operatorname{Re}_{c}=313.8$ whereas the other bifurcation is found at $\operatorname{Re}_{c}=313.3$. These 2 critical values are really closed to each other, but modes and flows on post-bifurcated branches are clearly different (see next subsection). Using a finer mesh, the spanwise bifurcation is found early at $\operatorname{Re}_{c}=308.3$ against $\operatorname{Re}_{c}=314.8$ for the other one.

In Table 5, the fourth and fifth primary bifurcations are reported. The critical values found differ about $2 \%$ depending of the meshes used. bifurcation type is found at $\operatorname{Re}_{c}=568.8$ for $A_{i}=8$ and $\operatorname{Re}_{c}=518.7$ for $A_{i}=10$. However, for $A_{i}=9$, the detection of the bifurcation type failed with the criterion proposed in the work of Cochelin and Medale ${ }^{12}$ and retained for this study. Nevertheless a pole appears in the rational representation of the series (as used in the work of Guevel et $\mathrm{al}^{19}$ ) indicating a bifurcation for $\mathrm{Re}_{c}=545$. In this case, we were not able to determine the bifurcation type. Finer spatial discretization or finite element of higher degree might be needed to ensure a perfect detection.

Evolution of critical Reynolds number against the spanwise aspect ratio is plotted in Figure 10. The trend of the $\boldsymbol{\Delta}$ and bifurcation type is similar to that of the first primary bifurcation described in Section 4.2.3. As the body is narrow, which corresponds to small geometric ratio $A_{i}$, there is a stabilization effect on the bifurcation phenomenon. In the case of the spanwise breaking primary bifurcation $\square$, this stabilization effect occurs for higher ratio. The trend is not the same for this $\square$ primary bifurcation type. It might be explained by the fact that these kinds of bifurcation are comparable to a 2D bifurcation, whereas the spanwise symmetry-breaking primary bifurcation is a real 3D effect.

\subsubsection{Primary bifurcation modes}

The bifurcation types $\square, \mathbf{\Lambda}$, and are now described for the ratio $A_{i}=8$ (see Figures 11 and 12). Velocity components and pressure of bifurcation modes are proposed for the case $A_{i}=8$ using $L=55 \mathrm{~h}$.

The bifurcation modes $\boldsymbol{\Phi}_{\mathrm{PB} 3}(\boldsymbol{\Lambda})$ and $\boldsymbol{\Phi}_{\mathrm{PB} 4}(\diamond)$ are both similar in their topology with the first primary bifurcation $\boldsymbol{\Phi}_{\mathrm{PB} 1}$ seen in Figure 7. The bifurcation mode $\boldsymbol{\Phi}_{\mathrm{PB} 2}(\square)$, which breaks the spanwise symmetry, has a different structure. The latter is in perfect agreement with the mode described in the work of Medale and Cochelin. ${ }^{14}$

\subsection{Case with secondary bifurcation for $\boldsymbol{A}_{\boldsymbol{i}}=8$}

In previous subsections, only primary bifurcations were discussed. Now, results for the ratio $A_{i}=8$ with secondary bifurcation (SB) are presented. A secondary bifurcation appears on a nonfundamental post-bifurcated branch where a symmetry remains.

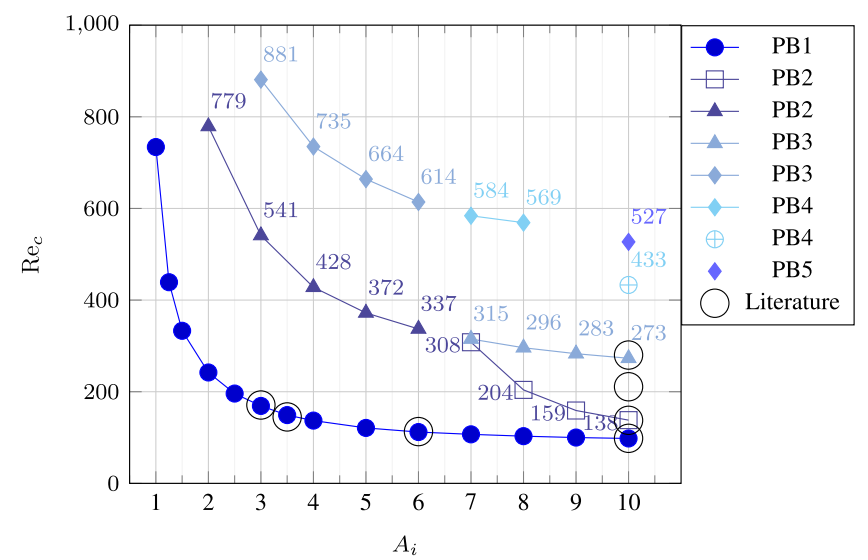

FIGURE 10 Critical Reynolds number associated to primary bifurcation for several $A_{i}$ using ELMER coupled with the asymptotic-numerical method. In black circles, values from the literature [Colour figure can be viewed at wileyonlinelibrary.com] 


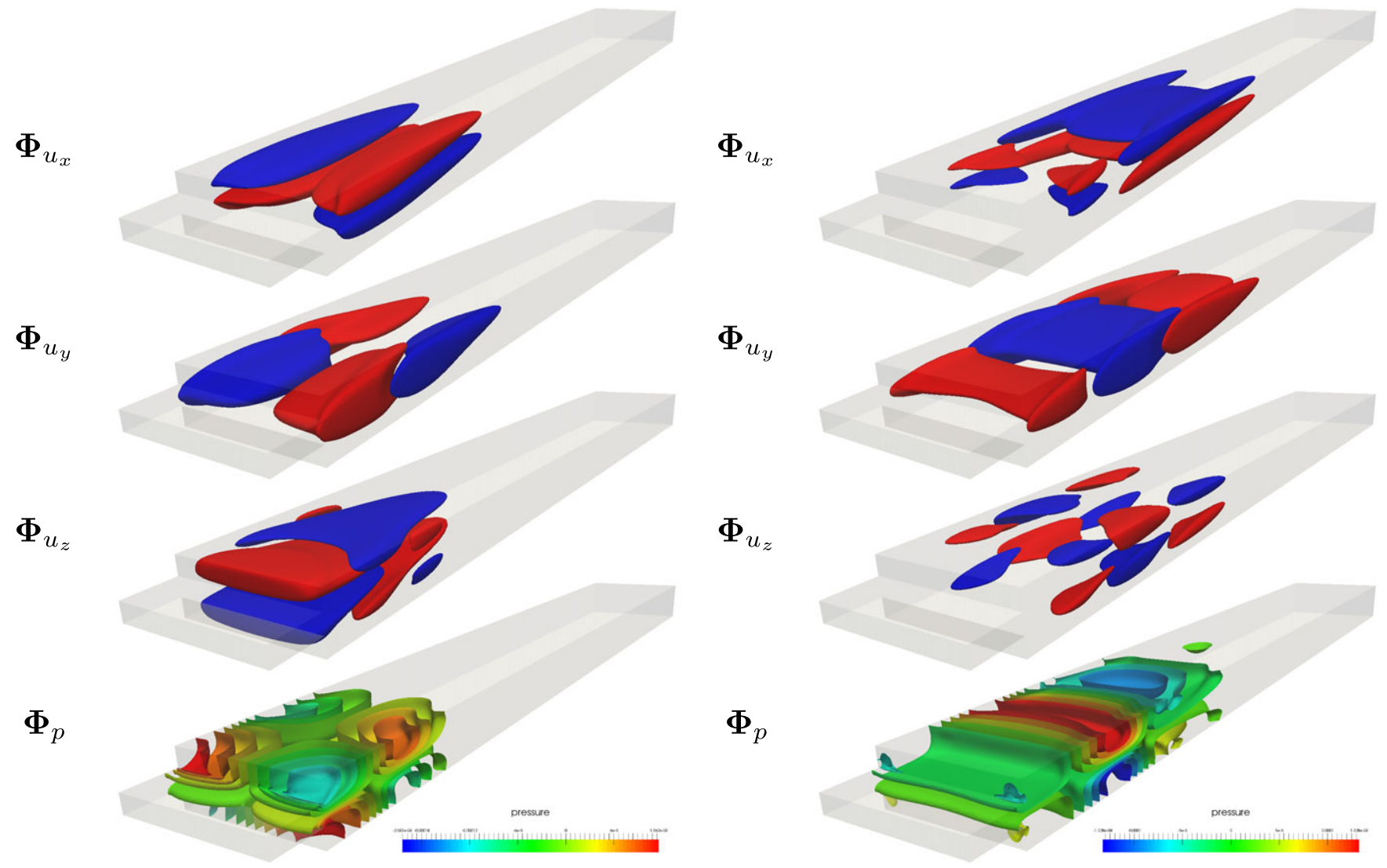

FIGURE 11 Bifurcation modes $\boldsymbol{\Phi}_{\mathrm{PB} 2} \square$ (Left) and $\boldsymbol{\Phi}_{\mathrm{PB} 3} \Delta$ (right). Velocity components and pressure for $E=3, A_{i}=8, L=55 h$. Contours for $\boldsymbol{\Phi}_{u}$ are $10^{-3} \cdot 10^{-4}, 10^{-4}$. Red: positive; blue: negative

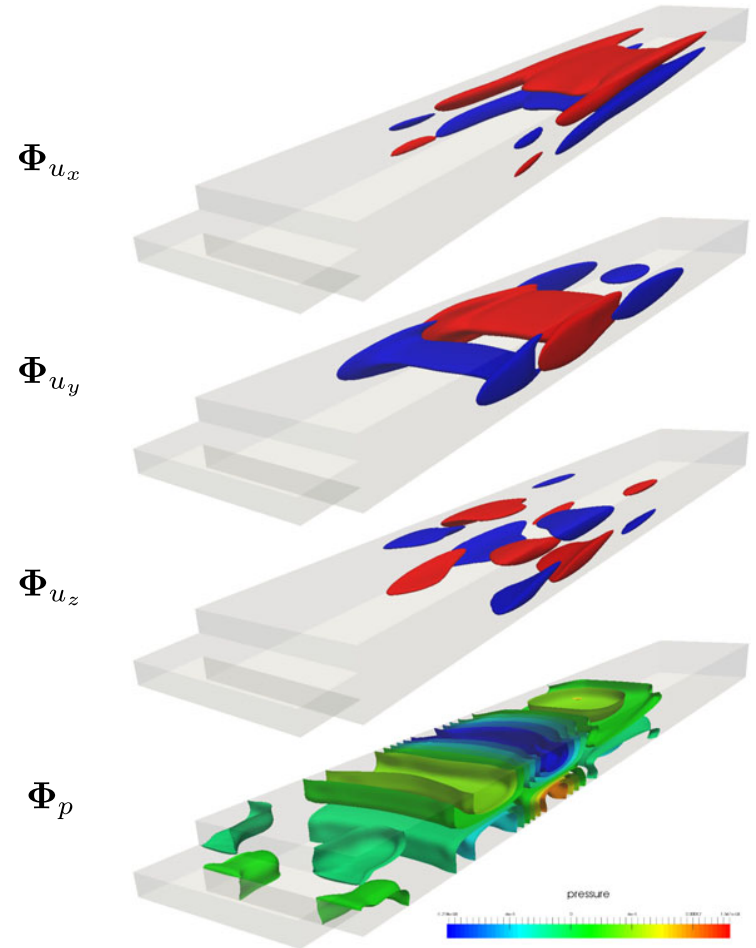

FIGURE 12 Bifurcation modes $\boldsymbol{\Phi}_{\mathrm{PB} 4}$ noted as $\downarrow$. Velocity components and pressure for $E=3, A_{i}=8, L=55 h$. Contours for $\boldsymbol{\Phi}_{u}$ are $10^{-3} \cdot 10^{-4}, 10^{-4}$. Red: positive; blue: negative 
TABLE 6 Critical Reynolds numbers for the steady bifurcations using finer or longer mesh with the ratio $A_{i}=8$. NS stands for not searched

\begin{tabular}{ccccc} 
& FB1 & FB2 & FB3 & FB4 \\
\hline PB & 102.36 & 203.87 & 297.59 & 569.01 \\
SB1 & 442.06 & 315.08 & 653.05 & NS \\
SB2 & 602.93 & 602.20 & NS & NS \\
\hline
\end{tabular}

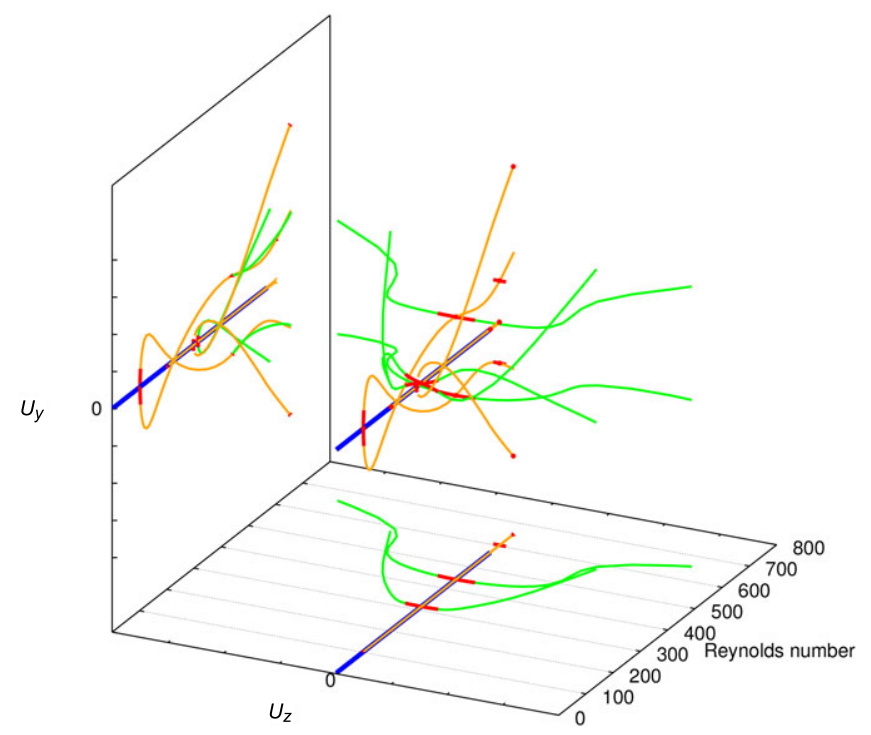

FIGURE 13 Three-dimensional bifurcation diagram obtained using the asymptotic-numerical method for the ratio $A_{i}=8$ using a fine and long mesh. Two distinct 2D bifurcation diagrams are also plotted as projections on the considered plane [Colour figure can be viewed at wileyonlinelibrary.com]

\subsection{1 | Critical Reynolds numbers}

In Table 6, the critical Reynolds number of primary and secondary bifurcations depending on the initial fundamental branch part as presented in Figure 2 is reported.

\subsection{2 । Bifurcation diagram}

A 3D bifurcation diagram is presented in Figure 13, where solution branches rely on $\mathbf{u}_{y}$ and $\mathbf{u}_{z}$ at probe as in Section 4.2.1. The first part of this diagram has been presented in Figure 2. The complexity of this diagram is clearly seen. Thus, a synthetic diagram is presented in Figure 14 with information on the state of symmetry of the branches. Nevertheless, the ANM-based method makes it possible to monitor the branch solutions and to perform branch switching very easily.

A remark is made for the second primary bifurcation, which breaks the spanwise symmetry, ${ }^{14,28}$ but does not appear on this diagram. This is due to the choice of the probe on the $\mathrm{X}$-axis. The flow is symmetric only with respect to the $\mathrm{X}$-axis so that both velocity components $\mathbf{u}_{y}$ and $\mathbf{u}_{z}$ at probe stay null.

This diagram is proposed in a synthetic version as presented in the work of Golubitsky and Schaeffer. ${ }^{38}$ It gathered both antisymmetric solutions (such as BBr1n and BBr1p) into one family branch. Moreover, the symmetry group remaining to be broken is recalled. For example, $\mathbb{Z}_{2}(z)$ is the planar symmetry regarding the vertical plane. In this case, the spanwise symmetry is still present. The double plane or X-axis symmetry is classically noted as $\mathbb{Z}_{2}(y) \oplus \mathbb{Z}_{2}(z)$. 


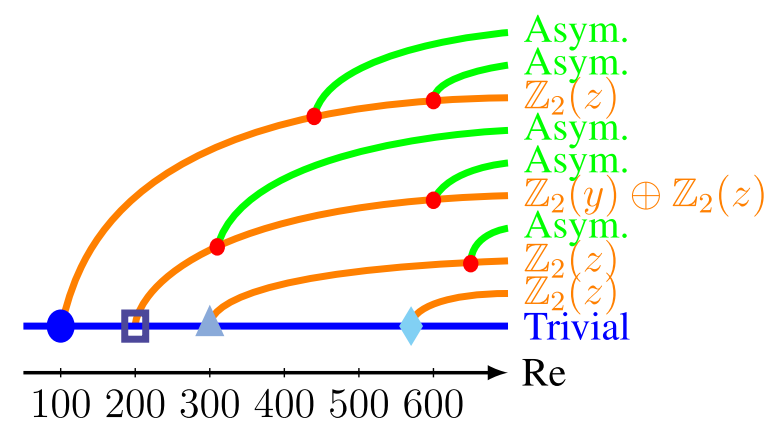

FIGURE 14 Synthetic bifurcation diagram. Critical Reynolds numbers are respected. Primary bifurcation type and symmetry state are given for each concerned branch
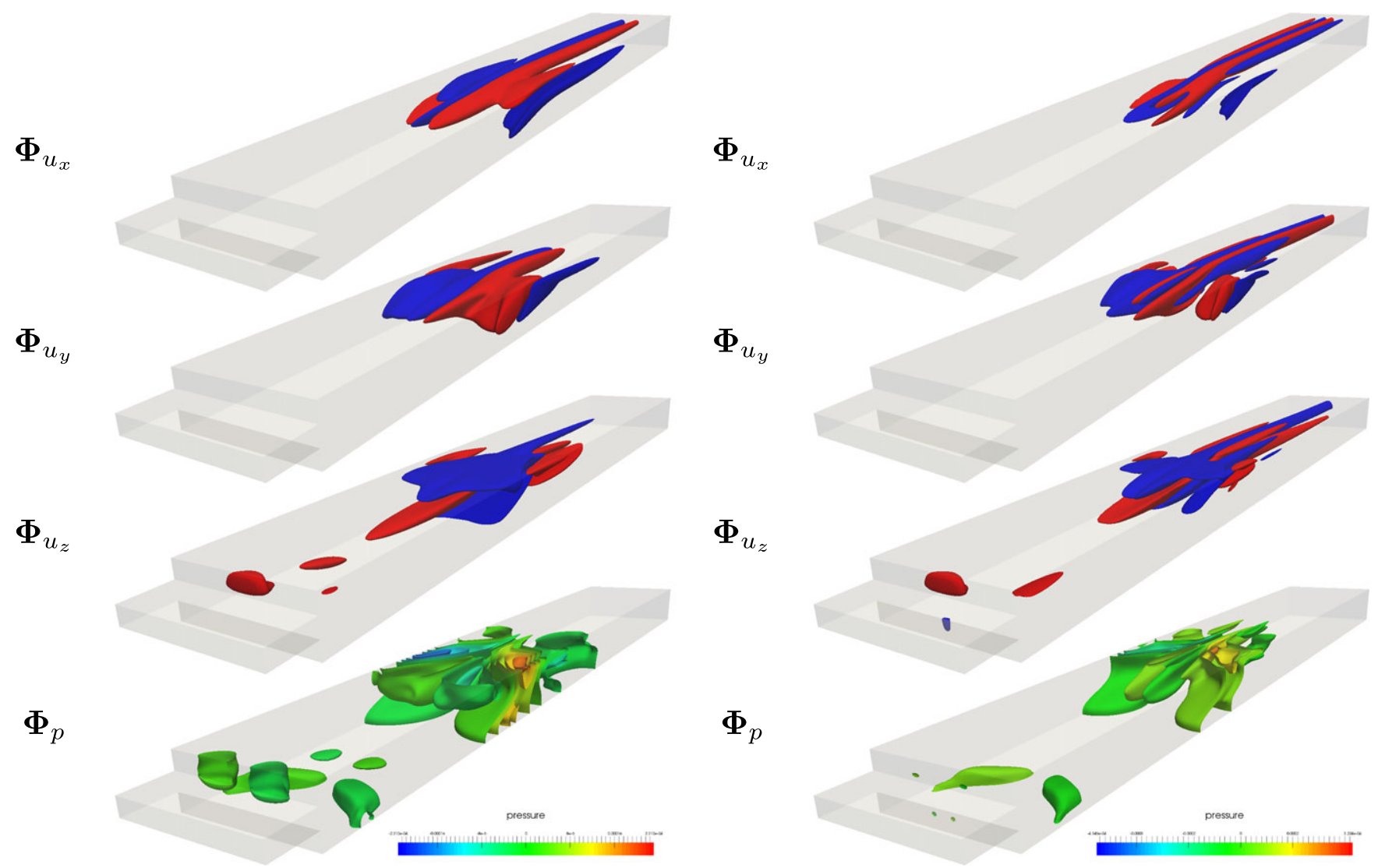

$\Phi_{u_{z}}$

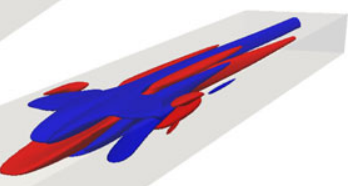

FIGURE 15 Secondary bifurcation modes $\boldsymbol{\Phi}_{\mathrm{PB} 1 \mathrm{SB} 1}$ (left) and $\boldsymbol{\Phi}_{\mathrm{PB} 1 \mathrm{SB} 2}$ (right). Velocity components and pressure for $E=3 A_{i}=8 L=55 h$. Contours for $\boldsymbol{\Phi}_{u}$ are $10^{-3} \cdot 10^{-4}, 10^{-4}$. Red: positive; blue: negative

\subsection{3 | Secondary bifurcation modes}

The secondary bifurcation modes are now described. Velocity components and pressure are proposed for the case $A_{i}=8$ using $L=55 h$. Those bifurcations lead to either a fully nonsymmetric flow, or let the previous symmetry unchanged after the bifurcation point.

In the case of the first primary bifurcation, the nontrivial post-bifurcated branch still conserves the spanwise symmetry. Both $\boldsymbol{\Phi}_{\mathrm{PB} 1 \mathrm{SB} 1}$ and $\boldsymbol{\Phi}_{\mathrm{PB} 1 \mathrm{SB} 2}$ in Figure 15 are similar in topology.

In the case of the second primary bifurcation, the nontrivial post-bifurcated branch still has a symmetry to be broken, ie, $\mathbb{Z}_{2}(y) \oplus \mathbb{Z}_{2}(z)$. Here, $\boldsymbol{\Phi}_{\mathrm{PB} 2 \mathrm{SB} 1}$ and $\boldsymbol{\Phi}_{\mathrm{PB} 2 \mathrm{SB} 2}$ in Figure 16 are not similar in topology. $\boldsymbol{\Phi}_{\mathrm{PB} 2 \mathrm{SB} 1}$ looks like $\boldsymbol{\Phi}_{\mathrm{PB} 3}$ as a top-bottom symmetry-breaking bifurcation mode.

The last secondary bifurcation mode $\boldsymbol{\Phi}_{\mathrm{PB} 3 \mathrm{SB} 1}$ in Figure 17 has a complex structure without well-defined features. 


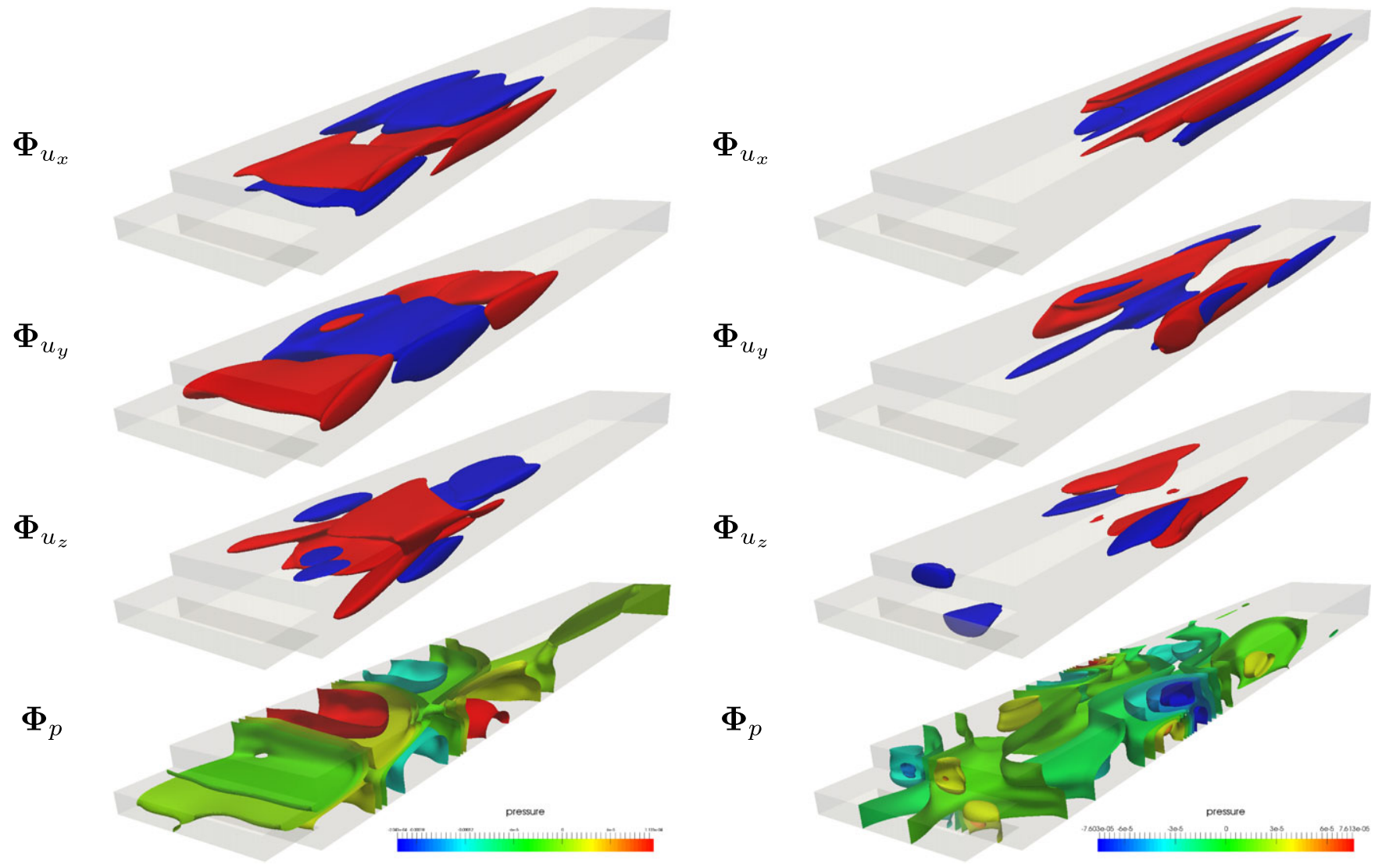

FIGURE 16 Secondary bifurcation modes $\boldsymbol{\Phi}_{\mathrm{PB} 2 \mathrm{SB} 1}$ (left) and $\boldsymbol{\Phi}_{\mathrm{PB} 2 \mathrm{SB} 2}$ (right). Velocity components and pressure for $E=3 A_{i}=8 L=55 h$. Contours for $\boldsymbol{\Phi}_{u}$ are $10^{-3} \cdot 10^{-4}, 10^{-4}$. Red: positive; blue: negative

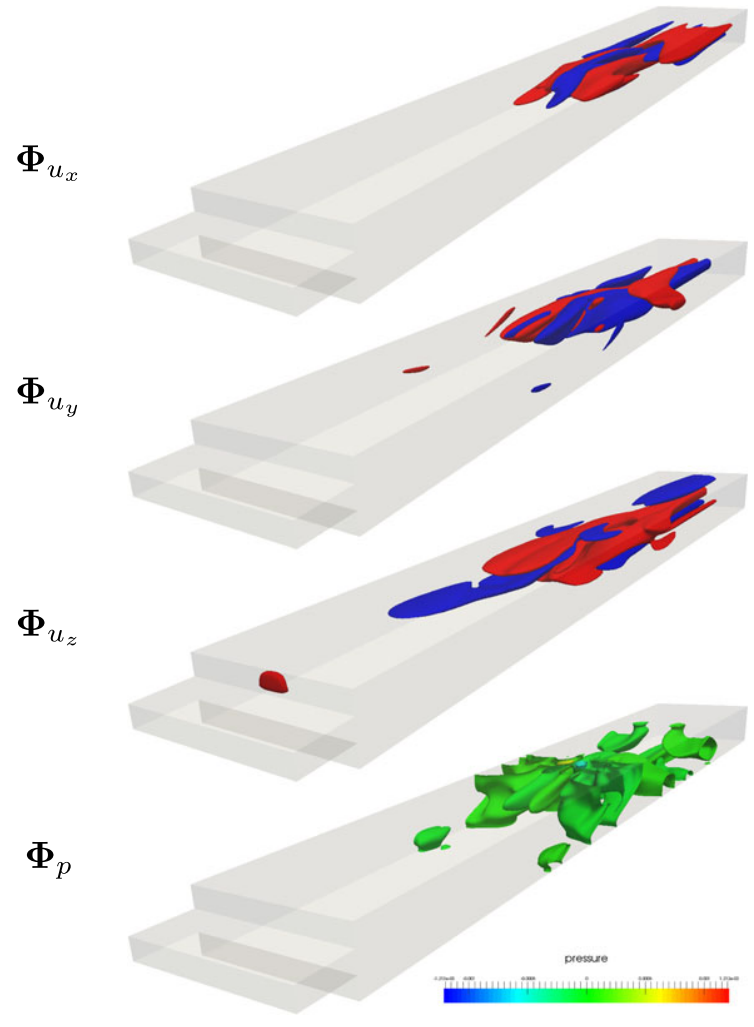

FIGURE 17 Secondary bifurcation mode $\boldsymbol{\Phi}_{P B 3 S B 1}$. Velocity components and pressure for $E=3 A_{i}=8 L=55 h$. Contours for $\boldsymbol{\Phi}_{u}$ are $10^{-3} \cdot 10^{-4}, 10^{-4}$. Red: positive; blue: negative 


\section{5 | CONCLUSIONS}

A numerical bifurcation analysis for the 3D sudden expansion has been proposed. Continuation of steady flow solutions, bifurcation detection, and branch-switching techniques are implemented using the ANM. Power series analysis makes it possible to accurately detect bifurcation points. Specific continuation technique is used in the case of the path following of postcritical branch of steady flow solutions. These techniques are implemented as new modules and user-defined solver in ELMER. Implementation of MUMPS as serial direct linear solver using the multithread features of OpenBlas allows us to perform 3D flow simulation up to 9.7 million degrees of freedom in this present study. Large-scale problems are now possibly studied, either only for steady flow continuation or for a detailed bifurcation analysis.

Three-dimensional sudden expansion critical values and flow topologies are correctly reproduced. The critical Reynolds numbers and modes for bifurcation are in good agreement with the literature. The proposed simplified branch-switching method at a pitchfork bifurcation is efficient. The stabilized FEM used in ELMER does not perturb the bifurcation analysis. Furthermore, while previous ANM studies ${ }^{12,14,15,17-21,30}$ did not use pressure information because static condensation was used, pressure component is successfully used in this work within the ANM bifurcation analysis framework. As full velocity-pressure mixed vectors are used, flows and specific vectors are presented in this study with pressure information.

New results are obtained. Firstly, evolution of the critical Reynolds number for several primary bifurcations has been determined for aspect ratio $A_{i}$ from 1 to 10 . Those primary bifurcations are strongly stabilized with small spanwise aspect ratios. Different kinds of bifurcation were tracked and depicted. Secondly, we determined that the spanwise bifurcation described in the works of Medale and Cochelin ${ }^{14}$ and Chiang et $\mathrm{al}^{28}$ exists for ratios $A_{i} \geq 7$. Thirdly, a critical Reynolds number is found at $\mathrm{Re}_{c \mathrm{~PB} 1}=734$ for the aspect ratio $A_{i}=1$. This was not found in the work of Tsui and Wang $^{29}$ for $R e \leq 300$. To the best knowledge of the authors, this critical value was not previously reported in the literature. Finally, a bifurcation diagram and description of the primary and secondary bifurcation modes were proposed for the case $A_{i}=8$.

A generic tool is available for large-scale bifurcation analysis of the 3D Navier-Stokes equations. Eigensolver might be used in ELMER to determine the stability of flow solutions. Additional techniques based on the ANM may now be implemented, such as parametric analysis of steady bifurcation using homotopy ${ }_{15}^{15}$ bifurcation analysis of non-Newtonian fluids, ${ }^{20}$ Hopf bifurcation detection, ${ }^{18,52-55}$ and transient nonlinear solvers. ${ }^{13,56}$ It is also challenging to study multiple bifurcations or to couple the reduced-order model techniques with the ANM-based methods for 3D flows.

Lastly, the series analysis detection method is based on the properties of singular tangent operators during a continuation procedure. Then, it is expected to be able to detect Hopf bifurcation using a specific continuation technique.

\section{ACKNOWLEDGMENTS}

The authors would like to thank Obame Endamne Sylvain for the linear solvers comparisons proposed in Appendix A.1.

\section{ORCID}

Y. Guevel (iD http://orcid.org/0000-0001-8931-0168

\section{REFERENCES}

1. Dijkstra HA, Wubs FW, Cliffe AK, et al. Numerical bifurcation methods and their application to fluid dynamics: analysis beyond simulation. Commun Comput Phys. 2014;15:1-45.

2. Dhooge A, Govaerts W, Kuznetsov YA. MATCONT: a MATLAB package for numerical bifurcation analysis of ODES. ACM Trans Math Softw (TOMS). 2003;29(2):141-164.

3. Doedel EJ, Wang X. AUTO 94: Software for Continuation and Bifurcation Problems in Ordinary Differential Equations. Pasadena, CA: California Institute of Technology, Department of Applied Mathematics; 1994.

4. Kłosiewicz P, Broeckhove J, Vanroose W. Using pseudo-arclength continuation to trace the resonances of the Schrödinger equation. Comput Phys Commun. 2009;180(4):545-548. Special issue based on the Conference on Computational Physics 2008 (CCP 2008).

5. Salinger AG, Bou-Rabee NM, Pawlowski RP, et al. LOCA 1.0 Library of continuation algorithms: Theory and implementation manual. [Technical Report SAND2002-0396]. Albuquerque, New Mexico and Livermore, CA: Sandia National Laboratory; 2002.

6. The Trilinos Project. https://trilinos.org/. 
7. Winters K. Bifurcation and stability: a computational approach. Comput Phys Commun. 1991;65(1-3):299-309.

8. Doedel E, Keller HB, Kernevez JP. Numerical analysis and control of bifurcation problems (I): Bifurcation in finite dimensions. Int J Bifurc Chaos. 1991;1(3):493-520.

9. Seydel R. Practical Bifurcation and Stability Analysis. Interdisciplinary Applied Mathematics. New York, NY: Springer Verlag; 2009.

10. Cochelin B, Damil N, Potier-Ferry M. Méthode Asymptotique Numérique. Collection méthodes numériques. Paris, France: Hermès Science Publications; 2007.

11. Bekhoucha F, Rechak F, Duigou L, Cadou JM. Branch switching at Hopf bifurcation analysis via asymptotic numerical method: application to nonlinear free vibrations of rotating beams. Commun Nonlinear Sci Numer Simul. 2015;22(1-3):713-730.

12. Cochelin B, Medale M. Power series analysis as a major breakthrough to improve the efficiency of asymptotic numerical method in the vicinity of bifurcations. J Comput Phys. 2013;236:594-607.

13. Guevel Y, Girault G, Cadou JM. Numerical comparisons of high-order nonlinear solvers for the transient Navier-Stokes equations based on homotopy and perturbation techniques. J Comput Appl Math. 2015;289:356-370. Sixth International Conference on Advanced Computational Methods in Engineering (ACOMEN 2014).

14. Medale M, Cochelin B. High performance computations of steady-state bifurcations in 3D incompressible fluid flows by asymptotic numerical method. J Comput Phys. 2015;299:581-596.

15. Guevel Y, Girault G, Cadou JM. Parametric analysis of steady bifurcations in 2D incompressible viscous flow with high order algorithm. Comput Fluids. 2014;100:185-195.

16. Cochelin B. A path-following technique via an asymptotic-numerical method. Comput Struct. 1994;53(5):1181-1192.

17. Cadou JM, Potier-Ferry M, Cochelin B, Damil N. ANM for stationary Navier-Stokes equations and with Petrov-Galerkin formulation. Int J Numer Methods Eng. 2001;50(4):825-845.

18. Cadou JM, Potier-Ferry M, Cochelin B. A numerical method for the computation of bifurcation points in fluid mechanics. Eur J Mech B/Fluids. 2006;25(2):234-254.

19. Guevel Y, Boutyour H, Cadou JM. Automatic detection and branch switching methods for steady bifurcation in fluid mechanics. J Comput Phys. 2011;230(9):3614-3629.

20. Jawadi A, Boutyour H, Cadou JM. Asymptotic numerical method for steady flow of power-law fluids. J Non-Newtonian Fluid Mech. 2013;202:22-31.

21. Medale M, Cochelin B. A parallel computer implementation of the asymptotic numerical method to study thermal convection instabilities. J Comput Phys. 2009;228(22):8249-8262.

22. Durst F, Melling A, Whitelaw JH. Low Reynolds number flow over a plane symmetric sudden expansion. J Fluid Mech. 1974;64:111-128.

23. Fearn RM, Mullin T, Cliffe KA. Nonlinear flow phenomena in a symmetric sudden expansion. J Fluid Mech. 1990;211:595-608.

24. Baloch A, Townsend P, Webster M. On two- and three-dimensional expansion flows. Comput Fluids. 1995;24(8):863-882.

25. Battaglia F, Papadopoulos G. Bifurcation characteristics of flows in rectangular sudden expansion channels. J Fluids Eng. 2005;128(4):671-679.

26. Schreck E, Schäfer M. Numerical study of bifurcation in three-dimensional sudden channel expansions. Comput Fluids. 2000;29(5):583-593.

27. Chiang T, Sheu TW, Wang S. Side wall effects on the structure of laminar flow over a plane-symmetric sudden expansion. Comput Fluids. 2000;29(5):467-492.

28. Chiang TP, Sheu TW, Hwang RR, Sau A. Spanwise bifurcation in plane-symmetric sudden-expansion flows. Phys Rev E. 2002;65(1):016306.

29. Tsui YY, Wang HW. Side-wall effects on the bifurcation of the flow through a sudden expansion. Int J Numer Methods Fluids. 2008;56(2):167-184.

30. Cadou JM, Guevel Y, Girault G. Numerical tools for the stability analysis of 2D flows: application to the two- and four-sided lid-driven cavity. Fluid Dyn Res. 2012;44(3):031403.

31. Cadou JM, Potier-Ferry M. A solver combining reduced basis and convergence acceleration with applications to non-linear elasticity. Int J Numer Method Biomed Eng. 2010;26(12):1604-1617.

32. Van Dyke M. Analysis and improvement of pertubation series. Q J Mech Appl Math. 1974;27(4):423-450.

33. Keller HB. Numerical solution of bifurcation and nonlinear eigenvalue problems. In: Rabinowitz PH, ed. Applications of Bifurcation Theory. Cambridge, MA: Academic Press; 1977:359-384.

34. Brezzi F, Rappaz J, Raviart PA. Finite dimensional approximation of nonlinear problems part III: simple bifurcation points. Numer Math. 1982;38(1):1-30.

35. Vannucci P, Cochelin B, Damil N, Potier-Ferry M. An asymptotic-numerical method to compute bifurcating branches. Int J Numer Methods Eng. 1998;41(8):1365-1389.

36. Boutyour EH, Zahrouni H, Potier-Ferry M, Boudi M. Bifurcation points and bifurcated branches by an asymptotic numerical method and Padé approximants. Int J Numer Methods Eng. 2004;60(12):1987-2012.

37. Vanderbauwhede A. Lyapunov-Schmidt method for dynamical systems. In: Meyers RA, ed. Mathematics of Complexity and Dynamical Systems. New York, NY: Springer; 2011:937-952.

38. Golubitsky M, Schaeffer DG. Singularities and Groups in Bifurcation Theory. Vol I. New York, NY: Springer Verlag; 1984.

39. Werner B, Spence A. The computation of symmetry-breaking bifurcation points. J Numer Anal. 1984;21(2):388-399.

40. CSC - IT Center for Science. ELMER - Finite Element Solver for Multiphysical Problems; 1995. www.csc.fi/elmer 
41. Amestoy PR, Duff IS, L’Excellent JY, Koster J. A fully asynchronous multifrontal solver using distributed dynamic scheduling. SIAM J Matrix Anal Appl. 2001;23(1):15-41.

42. Xianyi Z, Wang Qian ZY. Model-driven level 3 BLAS performance optimization on Loongson 3A processor. Paper presented at: 2012 IEEE 18th International Conference on Parallel and Distributed Systems (ICPADS). 2012; Singapore.

43. Zienkiewicz O, Taylor R. The Finite Element Method, Fluid Dynamics. London, UK: Wiley; 2000.

44. Shah R, London A. Laminar flow forced convection in ducts: a source book for compact heat exchanger analytical data. Advances in Heat Transfer: Supplement. Cambridge, MA: Academic Press; 1978.

45. Titarev VA, Shakhov EM. Kinetic analysis of the isothermal flow in a long rectangular microchannel. Comput Math Math Phys. 2010;50:1221-1237.

46. Theofilis V, Duck P, Owen J. Viscous linear stability analysis of rectangular duct and cavity flows. J Fluid Mech. 2004;505:249-286.

47. Nicolas X, Luijkx JM, Platten JK. Linear stability of mixed convection flows in horizontal rectangular channels of finite transversal extension heated from below. Int J Heat Mass Transf. 2000;43(4):589-610.

48. Spiga M, Morino G. A symmetric solution for velocity profile in laminar flow through rectangular ducts. Int Commun Heat Mass Transfer. 1994;21(4):469-475.

49. Tanyeri M, Ranka M, Sittipolkul N, Schroeder CM. A microfluidic-based hydrodynamic trap: design and implementation. Lab Chip. 2011;11:1786-1794.

50. Délery J. Skin Friction Lines Pattern and Critical Points. Hoboken, NJ: John Wiley and Sons, Inc; 2013;1-26.

51. Battaglia F, Tavener SJ, Kulkarni AK, Merkle C. Bifurcation of low Reynolds number flows in symmetric channels. $A I A A J$. 1997;35(1):99-105.

52. Marquet O, Sipp D, Jacquin L. Sensitivity analysis and passive control of cylinder flow. J Fluid Mech. 2008;615:221-252.

53. Brezillon A, Girault G, Cadou JM. A numerical algorithm coupling a bifurcating indicator and a direct method for the computation of Hopf bifurcation points in fluid mechanics. Comput Fluids. 2010;39(7):1226-1240.

54. Girault G, Guevel Y, Cadou JM. An algorithm for the computation of multiple Hopf bifurcation points based on Padé approximants. Int J Numer Methods Fluids. 2012;68(9):1189-1206.

55. Heyman J, Girault G, Guevel Y, Allery C, Hamdouni A, Cadou JM. Computation of Hopf bifurcations coupling reduced order models and the asymptotic numerical method. Comput Fluids. 2013;76:73-85.

56. Razafindralandy D, Hamdouni A. Time integration algorithm based on divergent series resummation, for ordinary and partial differential equations. J Comput Phys. 2013;236:56-73.

57. Davis TA. Algorithm 832: UMFPACK v4.3-an unsymmetric-pattern multifrontal method. ACM Trans Math Softw. 2004;30(2):196-199.

58. Saad Y, Schultz MH. GMRES: a generalized minimal residual algorithm for solving nonsymmetric linear systems. SIAM J Sci Stat Comput. 1986;7(3):856-869.

59. Sonneveld P. CGS: a fast Lanczos-type solver for nonsymmetric linear systems. SIAM J Sci Stat Comput. 1989;10(1):36-52.

60. Van der Vorst HA. Bi-CGSTAB: a fast and smoothly converging variant of Bi-CG for the solution of nonsymmetric linear systems. SIAM J Sci Stat Comput. 1992;13(2):631-644.

61. Roland W. A transpose-free quasi-minimal residual algorithm for non-Hermitian linear systems. SIAM J Sci Comput. 1993;14(2):470-482.

62. Eisenstat SC, Elman HC, Schultz MH. Variational iterative methods for nonsymmetric systems of linear equations. SIAM J Numer Anal. 1983;20(2):345-357.

63. Saad Y. ILUT: a dual threshold incomplete LU factorization. Numer Linear Algebra Appl. 1994;1(4):387-402.

\section{APPENDIX A : LINEAR SOLVERS COMPARISONS}

In a context of large-scale problems analysis, question arises to use the most appropriate solver. It is known that iterative solvers are very efficient and computationally cheap compared with direct solvers. Nevertheless, it is briefly shown that ANM-based methods are tailored for direct solver because a sequence of algebraic systems is solved using the same matrix operator. Thus, a short comparison of direct and iterative linear solvers is proposed in this section. In the software ELMER FEM, the following linear solvers have been tested:

- Direct: $\mathrm{UMFPACK}^{57}$ and MUMPS ${ }^{41}$ 
- Iterative: GMRES $,^{58} \mathrm{CGS},{ }^{59} \mathrm{BiCGStab},{ }^{60} \mathrm{TFQMR},{ }^{61} \mathrm{GCR},{ }^{62}$ using the following iterative preconditioners: $I L U(n)$ with $n \in[0,9]$ and $I L U t^{63}$

\section{A.1 | Linear solvers' behavior}

Firstly, comparisons have been performed using a 2D sudden expansion. Same definitions of the geometric parameters presented for the 3D test case have been used. Hence, the following geometric features are: $h=10, E=3, l=3 h, L=30 h$ (see also the work of Guevel et $\mathrm{al}^{19}$ ). A Q4/4C mixed finite element ${ }^{43}$ has been used in ELMER. The ANM parameters are $N=30$ and $\eta=10^{-14}$. The same trivial initial solution at $\mathrm{Re}=0$ has been used. The ANM continuation procedure was stopped at $\operatorname{Re} \approx 81$.

\section{A.1.1 | Direct linear solvers}

A simple continuation test has been performed to compare 2 direct linear solvers available in ELMER. The number of ANM steps and total CPU time are recalled in Table A1. In our study, MUMPS is faster than UMFPACK.

\section{A.1.2 | Iterative linear solvers}

All combinations between the 5 iterative linear solvers, ie, GMRES, CGS, BiCGStab, TFQMR, GCR, and 11 preconditioners, ie, $I L U_{n}$ with $n \in[0,9] / I L U_{t}$, have been tested for one ANM step with a linear iteration tolerance parameter $T O L=10^{-8}$. The following conclusions have been reached: BiCGStab and CGS using either ILU(5) or ILUt were the most efficient in term of computational time.

Then, a simple ANM continuation procedure has been performed to compare those 2 iterative linear solvers using either ILU(5) or ILUt. Results are recalled in Table A2.

Using ILU(5) the number of linear iteration per ANM step grows from 3 iterations, for the first step, to 14 iterations when reaching $\mathrm{Re}>70$. Twelve ANM steps are necessary to reach $\mathrm{Re} \approx 81$ with a time per step between 20 to 60 seconds. In this case, BiCGStab+ILU(5) was the most efficient combination.

When using ILUt, only one linear iteration per ANM step is performed for both BiCGStab and CGS linear solvers. Seven ANM steps are performed to reach the Reynolds number $\operatorname{Re} \approx 81$ with approximately 16.5 seconds per ANM step. In this case, results are comparable for both combinations.

TABLE A1 Direct linear solvers

comparison using the

asymptotic-numerical method

(ANM) continuation procedure

between

$\mathrm{Re}=0$ and $\mathrm{Re} \approx 81$ in the case of $\mathrm{a}$

2-dimensional sudden expansion

\begin{tabular}{lrl} 
Package & ANM steps & Time \\
\hline MUMPS & 7 & $65.7 \mathrm{~s}$ \\
UMFPACK & 7 & $90.2 \mathrm{~s}$ \\
\hline
\end{tabular}

TABLE A2 Iterative linear solvers comparison using the asymptotic-numerical method (ANM) continuation procedure between $R e=0$ and $R e \approx 81$ in the case of a 2-dimensional sudden expansion using 2 types of preconditioner

\begin{tabular}{lrrr} 
Linear Solver & linear Iteration & ANM steps & Time \\
\hline BiCGStab+ILU(5) & {$[3,13]$} & 12 & $400.5 \mathrm{~s}$ \\
CGS+ILU(5) & {$[3,14]$} & 12 & $452.6 \mathrm{~s}$ \\
BiCGStab+ILUt & 1 & 7 & $114.6 \mathrm{~s}$ \\
CGS+ILUt & 1 & 7 & $115.4 \mathrm{~s}$
\end{tabular}


TABLE A3 CPU time in seconds and memory usage to perform one ANM step using truncation order $N=20$ for a 2-dimensional test case. Direct solver is MUMPS v4 with one thread. Iterative solver is BiCGStab with ILUt preconditioner with the notation [min, $\max$ ] for memory usage

\begin{tabular}{lrrrrr} 
& & \multicolumn{2}{c}{ MUMPS } & \multicolumn{2}{c}{ BiCGStab+ILUt } \\
Mesh & DOF & Time & RAM & Time & RAM \\
\hline 2D-Q4-M1 & 73155 & $1.4 \mathrm{~s}$ & $1.04 \mathrm{~GB}$ & $5.3 \mathrm{~s}$ & {$[0.21,0.97 \mathrm{~GB}]$} \\
2D-Q4-M2 & 289155 & $6.9 \mathrm{~s}$ & $3.69 \mathrm{~GB}$ & $56.8 \mathrm{~s}$ & {$[1.77,5.13 \mathrm{~GB}]$} \\
2D-Q4-M3 & 1149699 & $36.1 \mathrm{~s}$ & $14.62 \mathrm{~GB}$ & $721.1 \mathrm{~s}$ & {$[12.89,26.42 \mathrm{~GB}]$} \\
\hline
\end{tabular}

TABLE A4 CPU time in seconds to perform one ANM step using truncation order $N=20$ for a 3-dimensional test case. Direct solver is MUMPS v4 with one thread. Iterative solver is $\operatorname{BiCGStab}(l)$ using polynomial parameter $l=4$ with no preconditioner

\begin{tabular}{lrrrrr} 
& & \multicolumn{2}{c}{ MUMPS } & \multicolumn{2}{c}{ BiCGStab $(\boldsymbol{l}=4)$} \\
Mesh & DOF & Time & RAM & Time & RAM \\
\hline 3D-H8-M4 & 624100 & $524.7 \mathrm{~s}$ & $18.0 \mathrm{~GB}$ & $1865.8 \mathrm{~s}$ & $4.3 \mathrm{~GB}$ \\
3D-H8-M5 & 1197964 & $1673.9 \mathrm{~s}$ & $40.0 \mathrm{~GB}$ & $4716.2 \mathrm{~s}$ & $8.4 \mathrm{~GB}$ \\
3D-H8-M6 & 1584400 & $2979.5 \mathrm{~s}$ & $58.9 \mathrm{~GB}$ & $7305.7 \mathrm{~s}$ & $10.9 \mathrm{~GB}$ \\
\hline
\end{tabular}

\section{A.2 | Comparisons for 2D and 3D sudden expansions}

Combination of the iterative linear solver BiCGStab with precondtioner ILUt has been compared with the direct linear solver MUMPS. In the following, ANM truncation order has been set to $N=20$. Only one ANM step is under study.

Firstly, the same 2D geometry has been used with 3 mesh refinements. The CPU time and memory consumption are recalled in Table A3. The following 2 conclusions are made. MUMPS is faster. Moreover, the iterative process BiCGStab+ILUt uses approximately the same amount of memory than MUMPS.

Secondly, the flow in a 3D sudden expansion test case with the features $E=3, A_{i}=3, l=3 h, L=30 h$ has been studied with 3 mesh refinements. The $\mathrm{H} 8 / 8 \mathrm{C}$ mixed finite element ${ }^{43}$ has been used. An important remark is made regarding ILUt. It was not possible to perform all calculations with the combination BiCGStab+ILUt, whereas too much memory was required in ELMER. Thanks to the ELMER development team who advised us to use $\operatorname{BiCGStab}(l)$ with the parameter $l=4$. The CPU time and memory usage are recalled in Table A4. In conclusion, MUMPS is faster than $\operatorname{BiCGStab}(l=4)$ with no preconditioner, but more memory is required.

Some remarks are made regarding the choice of a linear solver for the ANM. The choice of the correct iterative linear solver and the corresponding best preconditioner or other parameters might depend on the part of the branch that is evaluated. A more precise study has to be performed in the future to give more detailed explanations on those peculiar behaviors. Lastly, it appeared that the multithreaded MUMPS direct linear solver used in this study made it possible to largely decrease the computational time regarding the iterative solvers available in ELMER. On the other side, a large amount of memory was required. 